\title{
Travelling Solitary Waves in the Discrete Schrödinger equation with Saturable nonlinearity: Existence, Stability and Dynamics
}

\author{
T.R.O. Melvin ${ }^{1}$, A.R. Champneys ${ }^{1}$, P.G. Kevrekidis ${ }^{2}$ and J. Cuevas ${ }^{3}$ \\ ${ }^{1}$ Department of Engineering Mathematics, University of Bristol, BS8 1TR, UK \\ ${ }^{2}$ Department of Mathematics and Statistics, University of Massachusetts, Amherst, \\ MA 01003-4515, USA \\ ${ }^{3}$ Grupo de Física No Lineal, Departamento de Física Aplicada I, \\ Escuela Universitaria Politécnica, C/ Virgen de África, 7, 41011 Sevilla, Spain
}

September 24, 2007

\begin{abstract}
The present work examines in detail the existence, stability and dynamics of travelling solitary waves in a Schrödinger lattice with saturable nonlinearity. After analysing the linear spectrum of the problem in the travelling wave frame, a pseudo-spectral numerical method is used to identify weakly non-local solitary waves. By finding zeros of an appropriately crafted tail condition, we can obtain the genuinely localised pulse-like solutions. Subsequent use of continuation methods allows us to obtain the relevant branches of solutions as a function of the system parameters, such as the frequency and inter-site coupling strength. We examine the stability of the solutions in two ways: both by imposing numerical perturbations and observing the solution dynamics, as well as by considering the solutions as fixed points of an appropriate map and computing the corresponding Floquet matrix and its eigenvalues. Both methods indicate that our solutions are robustly localised. Finally, the interactions of these solutions are examined in collision type phenomena, observing that relevant collisions are near-elastic, although they may, under appropriate conditions, lead to the generation of an additional pulse.
\end{abstract}

\section{Introduction}

In the past few years, there has been a large growth in the study of so called discrete solitons that is solitary waves and localised structures in spatially discrete media. This is to a large measure due to the development of numerous recent applications where such structures naturally arise and the corresponding models of differential-difference equations (DDEs), or so-called dynamical lattices, are relevant. Among these, one can mention the nonlinear optics of waveguide arrays [6, 22], the dynamics of Bose-Einstein condensates in periodic potentials [24], [29], [21], micro-mechanical models of cantilever arrays [39] or simple models of DNA [34]. One of the prototypical models that emerges in some form in all of these models (as a direct model, or as an envelope wave expansion or a tight binding approximation) is the so-called discrete nonlinear Schrödinger equation (DNLS) [20].

A particularly fruitful line of research has been the study of discrete localised structures in lattices that are optically induced within photorefractive crystals; see e.g., [13] for a recent review. The realisation of such two-dimensional periodic lattices, following the theoretical proposal of [10], allowed for the observation of discrete solitons [15, 27], as well as a wide array of additional coherent waveforms including, but not limited to, dipoles, quadrupoles, soliton trains, vector, necklace and ring solitons, see e.g. [44, 43, 30, 14]. Such crystals (of which strontium barium niobate (SBN) are the prototypical example) feature a, so-called, 
saturable nonlinearity. The coexistence of saturable nonlinearity with a periodic potential (induced by an ordinary polarization of light) for extraordinarily polarized probe beams in these media has naturally given rise to an interest in examining dynamical lattices of DNLS type, but with this saturable nonlinearity, rather than the standard cubic one $[18,40,25,7,26]$.

The class of DNLS models with saturable nonlinearity are also of particular interest in their own right, due to a feature first unveiled in [18]. In particular, it is well-known that in the standard DNLS model (see [20] and also references therein), there are two principal solitary wave solutions, the on-site and the inter-site soliton and the former has always lower energy than the latter. Hence, their energy difference, the celebrated Peierls-Nabarro (PN) barrier (a term coined from the theory of dislocations representing the energy that the dislocation needs to jump from a lattice site to the next) is always sign-definite. However, in the saturable DNLS model, this is no longer the case. In fact, the energy barrier changes sign and perhaps even more importantly can also precisely vanish at isolated points.

The vanishing of the PN barrier is not only of interest in regard to stationary solutions and their stability properties (see e.g., [28]), but importantly is associated with whether pulses can travel in such lattices without slowing down or shedding radiation. One may expect that near such points where the wave faces no potential barrier ${ }^{1}$, it is more natural to expect uninhibited travelling (although this idea is tempting, we will demonstrate below that it is, at least partially, problematic). The issue of undistorted travelling of exponentially localized excitations in DNLS type lattices has been important for a number of reasons. On the one hand, it is of particular physical relevance, since it would be desirable to transport optical (in the case of optical waveguides) or quantum (in the case of BECs in optical lattices) bits of information without radiative losses. On the other hand, it is an issue of mathematical delicacy and there has been some controversy on the existence (or not) of such solutions; see relevant details in $[37,9,12,1]$. The difficulty arises because seeking such travelling waves in the moving (with the speed of the wave) frame leads to so-called advance-delay equations which are hard to analyse. A recent negative result in this direction has come from the analysis of the so-called Salerno model [38], which is a homotopic interpolation between an integrable lattice (where travelling solutions exist in explicit form) and the non-integrable DNLS lattice. What was observed in $[16,17]$ was that as soon as the integrable limit was abandoned the travelling solutions developed tails and were no longer localized.

Our aim in the present paper, expanding on our earlier work of [28], is to settle this problem of travelling solutions for the saturable DNLS lattice, which, according to the above discussion appears as a more natural candidate to support travelling solitary waves. We examine the problem of existence of such waves, starting from the linear limit of the equation which will provide us with travelling periodic wave solutions, resonances with which we would in principle like to avoid. We then discuss our numerical method, which is based on a pseudo-spectral decomposition of the advance-delay equation due to [2] allied to a globally convergent Powell hybrid method. This tool used in combination with a tail condition to detect whether the tail of the wave is decaying or is oscillatory, allows us to identify the exponentially localized ones among our solutions. Finally, having obtained such solutions, we use continuation to follow their branches as a function of the system parameters (the inter-site coupling strength or the temporal frequency). We then discuss the stability of such solutions in two ways. Firstly, we dynamically integrate perturbed profiles of such solutions; what we observe typically in this case is that the solution remains localized but transforms itself into another solitary wave element on the same branch of solutions but with a different wavespeed and frequency. Secondly, whenever possible, we consider the solution as a fixed point of an appropriate map and obtain the corresponding Floquet matrix and the relevant Floquet multipliers which also indicate that our solutions are, in fact, dynamically stable. Finally, the dynamics of the solutions is also examined through

\footnotetext{
${ }^{1}$ Note a subtle assumption here: we are considering the more common situation, whereby the vanishing of the PN barrier implies the independence of the wave's energy on the location of its centre, although it is, in principle, possible that the PN energy barrier vanishes but the wave energy is still dependent on the location of the centre.
} 
direct simulations of their collisions and monitored through an appropriately defined coefficient of restitution associated with the collision (thinking of the waves as quasi-particles with appropriate momenta of their centres of mass). We find that the collisions of the waves are only very weakly inelastic, although occasionally such collisions may produce an additional solitary wave, a clear indication of the non-integrability of the model.

Our exposition will be structured as follows: section 2 contains the model and the analysis of its linear spectrum, while section 3 discusses our numerical method for obtaining the solutions. Section 4 presents an overview of the existence results, section 5 addresses the issue of stability and dynamical interactions (collisions) of travelling waves. Finally, section 6 summarizes our findings and presents some interesting directions for future study.

\section{Model Setup and Linear Analysis}

The DNLS with photorefractive nonlinearity is given by

$$
i \dot{u}_{n}(t)=-\varepsilon \Delta_{2} u_{n}(t)+\frac{\beta}{1+\left|u_{n}(t)\right|^{2}} u_{n}(t)
$$

where $\Delta_{2}$ is the standard second order spatial difference operator

$$
\Delta_{2} u_{n}(t)=u_{n+1}(t)-2 u_{n}(t)+u_{n-1}(t) .
$$

Eq. (1) is a Hamiltonian system

$$
E=\sum_{n}\left[\varepsilon\left|u_{n+1}-u_{n}\right|^{2}+\beta \log \left(1+\left|u_{n}\right|^{2}\right)\right] .
$$

It conserves the $l^{2}$-norm $P=\sum_{n}\left|u_{n}\right|^{2}$. The coefficient $\beta$ of the nonlinearity can be scaled to unity, and from now on we will take $\beta=1$. Study of the stationary modes of Eq.(1), using the transformation $u_{n}(t)=v_{n} e^{i \Lambda t}$ shows an interesting property of the saturable nonlinearity [28]. For a discrete set of parameter values the generalised Peierls-Nabarro energy barrier, $\Delta G=G_{o s}-G_{i s}$, where the subscripts os and $i s$ represent onsite and intersite modes respectively and $G=E-\Lambda P$, vanishes; see Figure 1 . The zeros where the energy barrier vanishes coincide with alterations in the stability of the onsite (i.e. centered on a lattice site) and intersite (i.e. centered between two lattice sites) stationary solitary wave modes. Therefore it could be imagined that genuinely localised travelling modes might bifurcate from these parameter values. Decreasing $\Lambda$ toward $\Lambda=0$ moves these zeros of $\Delta G$ closer to the anti-continuum limit, $\varepsilon=0$. For a fixed value of $\varepsilon=1$ the vanishings of the energy barrier for varying $\Lambda$ can be seen in Figure 1(b). As $\Lambda \rightarrow 1$ the amplitude of the stationary solutions grow exponentially and for $\Lambda \rightarrow 0$ the amplitude of the solutions goes to zero, and in these areas the calculation of the zeros is less accurate.

It is worth noting that for a true localised travelling wave to exist we would expect that the lattice would have to be translationally invariant. This implies that the energy barrier $\Delta G$ would have to be zero where the intersite mode has a maximum centred anywhere between two lattice sites and the onsite mode is centred on a lattice site. In fact the first zero of $\Delta G$, at $\varepsilon=\Lambda / 2$, is a true transparent point of the energy barrier. At this point the condition for stationary solutions to equation (1) reduces to $v_{n}=\varepsilon\left(v_{n+1}+v_{n-1}\right)\left(1+\left|v_{n}\right|^{2}\right)$. This is exactly the equation for stationary solutions of the Ablowitz-Ladik lattice, which is integrable and supports a two parameter family of travelling wave solutions. The transparent points computed in Figure 1 are only for a site centred mode and a symmetric intersite mode therefore $\Delta G$ may not be zero if an asymmetric intersite mode was used. However the existence of these points indicate that there is an increased possibility 

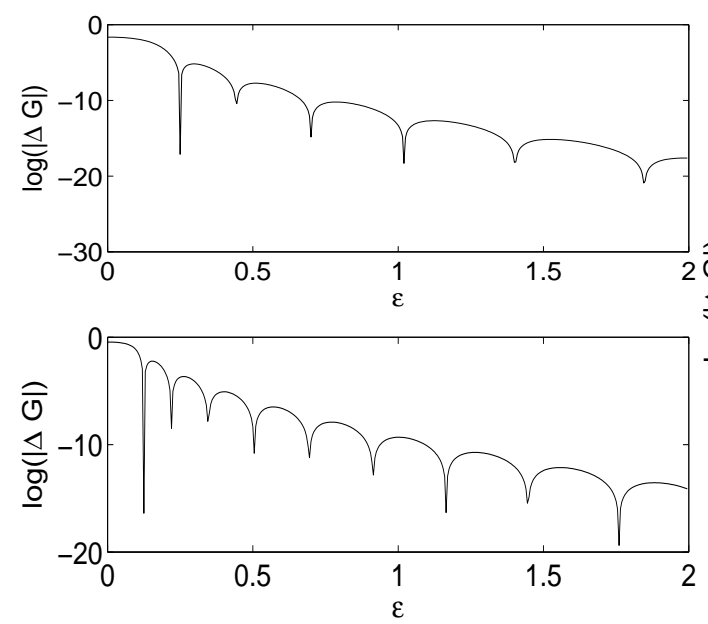

(a)

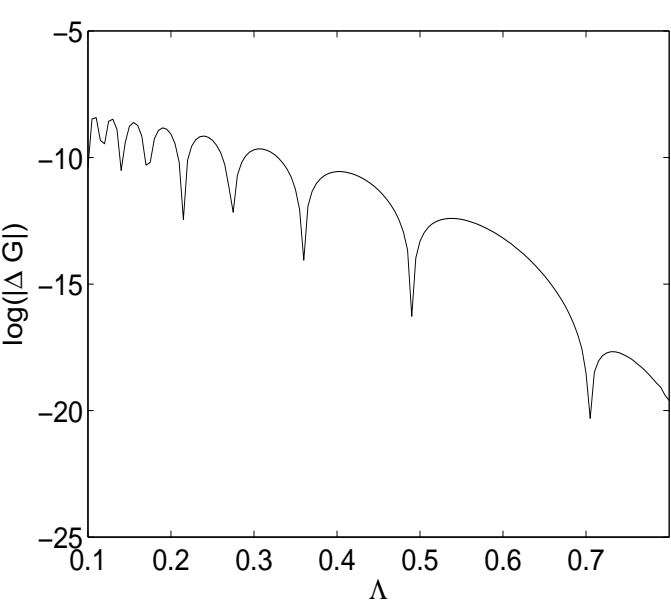

(b)

Figure 1: Energy difference $\Delta G$ between intersite and onsite modes of eq (1) for $\Lambda=0.5$, (a) top panel and $\Lambda=0.25$, (a) bottom panel. (b) The barrier is shown as a function of $\Lambda$ for fixed $\varepsilon=1$. Notice that the energy barrier vanishes for a discrete set of parameter values, which correspond to the points of stability alternation between the two modes.

of finding travelling wave solutions in the saturable discrete NLS over the cubic discrete NLS where the PN barrier is strictly non-zero.

Our main aim in what follows is to look for travelling wave solutions of the form

$$
u_{n}(t)=\psi(n-c t) e^{i(k n-\Lambda t)} .
$$

Using the substitution $z=n-c t$ with wave speed $c$, wavenumber $k$ and frequency $\Lambda$, we obtain a differential advance-delay equation in the travelling frame of reference $z$ moving with same wavespeed $c$ as the solution,

$$
-i c \psi^{\prime}(z)=(2 \varepsilon-\Lambda) \psi(z)-\varepsilon\left(\psi(z+1) e^{i k}+\psi(z-1) e^{-i k}\right)+\frac{1}{1+|\psi(z)|^{2}} \psi(z),
$$

where ' denotes differentiation with respect to $z$. Equation (3) is rotationally invariant, therefore the transformation

$$
\psi(z)=\tilde{\psi}(z) e^{-i k z}
$$

can be used to obtain

$$
-i c \psi^{\prime}(z)=(2 \varepsilon-\Lambda) \psi(z)-\varepsilon(\psi(z+1)+\psi(z-1))+\frac{1}{1+|\psi(z)|^{2}} \psi(z),
$$

where $\Lambda$ has been redefined as $\tilde{\Lambda}=k c+\Lambda$ and the tilde is dropped for convenience. Therefore $k=0$ can be taken in (3) without loss of generality and we assume that $\psi(0)$ is real.

We seek soliton solutions (homoclinic orbits to $\psi=0$ ) of equation (5). It is straightforward to show that (5) supports linear waves (radiation modes) that travel at the same wave speed $c$ as the sought for soliton. A so-called embedded soliton is obtained when the radiation mode component exactly vanishes in the tail of the solitary wave. At best, this can happen at a discrete set of values of the frequencies. An advance-delay equation of the form (5), can be seen as a dynamical system with an infinite-dimensional phase space [2]. Since such systems have a discrete spectrum only, then a necessary condition for codimension one solitons 


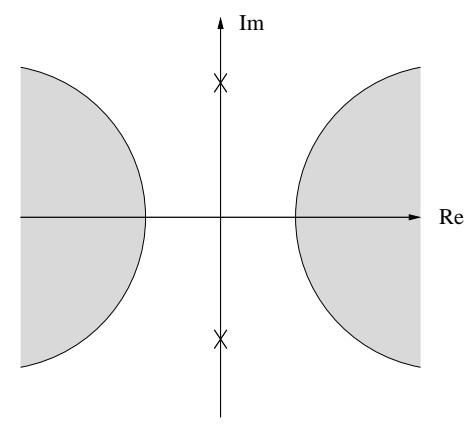

Figure 2: Condition of the spectrum of an advance delay equation for the existence of a codimension one embedded soliton. Shaded region indicates the area where the rest of the discrete spectrum is located.

is that the equilibrium has only one pair of eigenvalues on the imaginary axis; see the schematic of Figure 2. Therefore, to begin a search for embedded solitons, we look for parameter values where there are purely imaginary eigenvalues of the linear part of (5). To find such eigenvalues, we study the dispersion relation obtained by the substitution $\psi(z)=e^{i \lambda z}$ into the linear part of (5),

$$
c \lambda+(\Lambda-1)=-\varepsilon\left(e^{i \lambda}+e^{-i \lambda}-2\right)
$$

After rearrangement this can be written as

$$
c \lambda+(\Lambda-1)=4 \varepsilon \sin ^{2}\left(\frac{\lambda}{2}\right)
$$

We wish to investigate parameter regions where equation (7) has only one root $\lambda>0$. This is done using numerical continuation software to to follow points where an extra pair of roots is created. This is equivalent to solving (7) together with the condition for a double root

$$
c=2 \varepsilon \sin (\lambda) .
$$

The results are shown as dashed lines in Figure 3. The figure also depicts regions (spectral bands) where more than one pair of roots to (6) occurs. It can be seen that the spectral bands accumulate on the line $c=0$ so as $c$ is reduced toward zero then there is an ever increasing number of resonances. At the anti-continuum limit, $\varepsilon=0$, there are no resonances but the number of resonances increases as $\varepsilon$ is increased. Only the first few spectral bands are shown in Figure 3; hence, although it appears that the number of resonances begins to decrease again in the limit $c \rightarrow 0$, if more spectral bands had been shown we would observe that the number of resonances increases as $c$ is reduced toward $c=0$. The effects on the spectral bands of varying $\Lambda$ are shown in Figure 4, For $\Lambda=1$ (7) has odd symmetry and this is reflected in the spectral bands Figure 4(b) which now all reach $(0,0)$ and there is no longer any gap between the first and second spectral bands. Figure 4(a) shows the spectral bands for $\Lambda=0$ where the gap between the fundamental band and the second lower band has increased. The same analysis can be performed in the $(c, \Lambda)$ plane for fixed $\varepsilon$ giving Figure 5 .

Also of interest will be the decay rate of the solitary waves, this is governed by the real part of eigenvalues of the linear form of equation (5). Substituting $\psi(z)=e^{(\kappa+i \lambda) z}, \kappa, \lambda \in \mathbb{R}$, into (5) gives,

$$
\begin{aligned}
& c \kappa=2 \varepsilon \sin (\lambda) \sinh (\kappa), \\
& c \lambda=2 \varepsilon(1-\cos (\lambda) \cosh (\kappa))+1-\Lambda .
\end{aligned}
$$




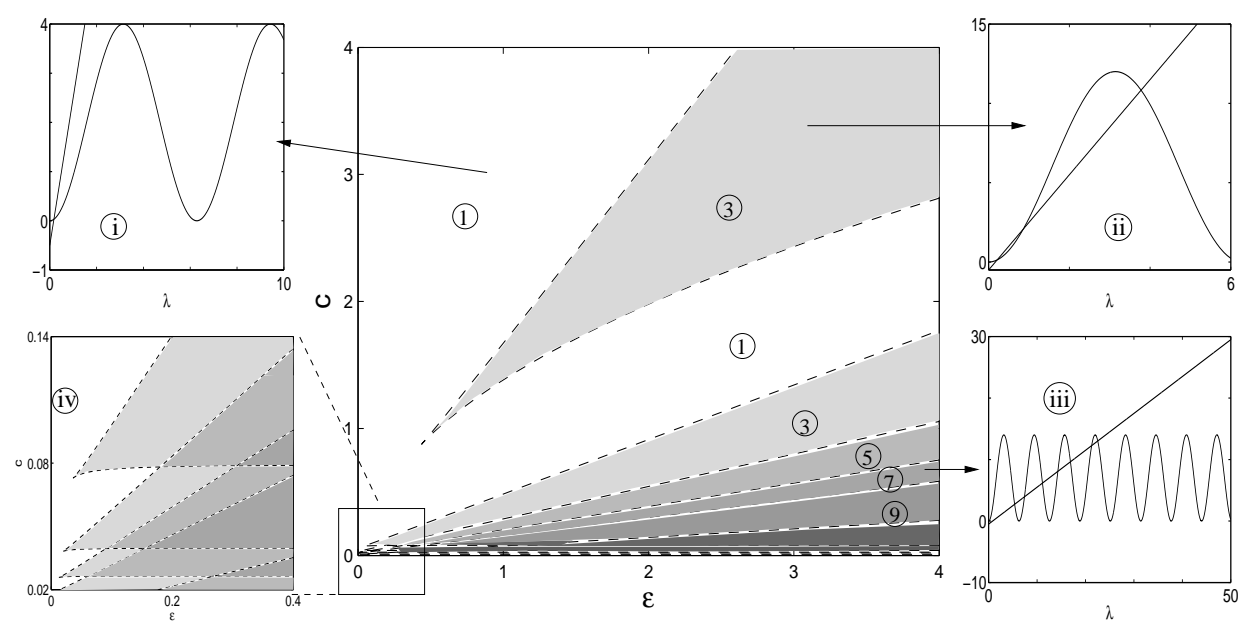

Figure 3: Intersections of (7) and (8) for $\Lambda=0.5$ and varying $c$ and $\varepsilon$. The shaded areas show regions where there is more than one branch of linear waves according to (7) \& (8). Values indicate the number of roots of (7) in each region. Subplots show left and right hand sides of $(7)$ for $(\varepsilon, c)=(1,3)$, one root (i), (3,3.5), three roots (ii) and (3.5,0.6), seven roots (iii). (iv) displays the overlapping of the bands for small $\varepsilon, c$, only the first six bands have been shown.



(a)

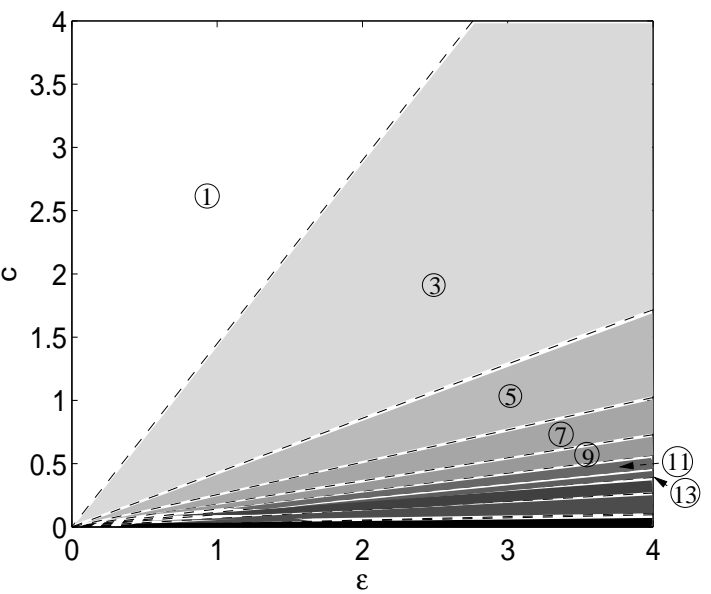

(b)

Figure 4: Intersections of (7) and (8) for (a) $\Lambda=0$, (b) $\Lambda=1$ and varying $c$ and $\varepsilon$. The shaded area shows where there is more than one branch of linear waves according to $(7) \&(8)$. Values indicate the number of roots of (7).

For travelling solutions $c \neq 0$ there are no purely real eigenvalues, $\lambda=0$, for $\Lambda \neq 1$ and only the zero eigenvalue, $\kappa=\lambda=0$ at $\Lambda=1$. The decay rate of solutions will be dominated by the terms with $\kappa$ closest to the imaginary axis. By rearranging equation (9), contours for fixed $\kappa$ of the fundamental spectral band $\lambda \in(0, \pi)$ can be plotted in parameter space, shown in Figure 6 . It is worth noting that the $\kappa=0$ contour is in fact the condition for purely imaginary roots and so overlays the spectral bands computed previously Figures 3-5. The eigenfunction corresponding to this fundamental band governs the localisation of the solution in the continumn limit. Therefore near to the $\kappa=0$ boundaries we would expect the width of any soliton to increase rapidly whilst the amplitude will go to zero as $\kappa \rightarrow 0$. 


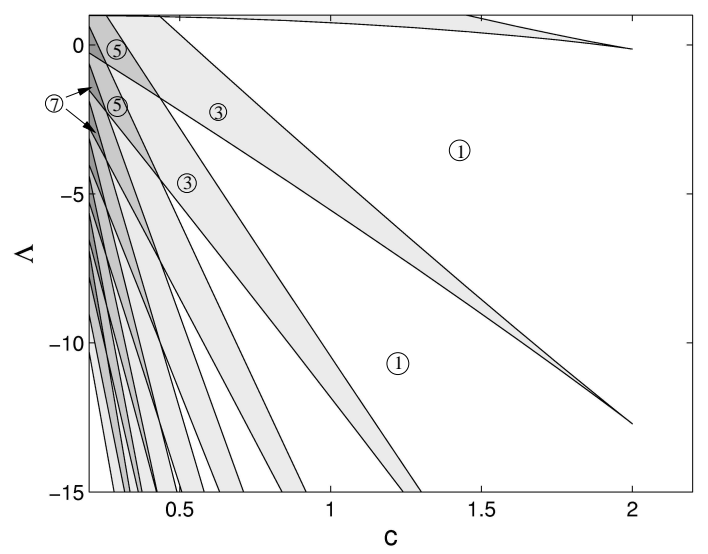

Figure 5: Analogue of Figure 3 for fixed $\varepsilon=1$ and varying $c$ and $\Lambda$. The shaded area shows where there is more than one branch of linear waves. Values indicate the number of purely imaginary eigenvalues.

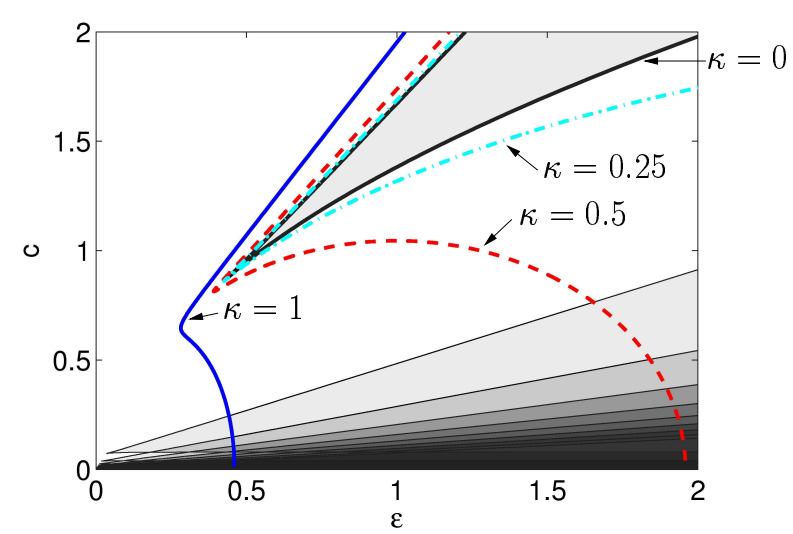

(a)

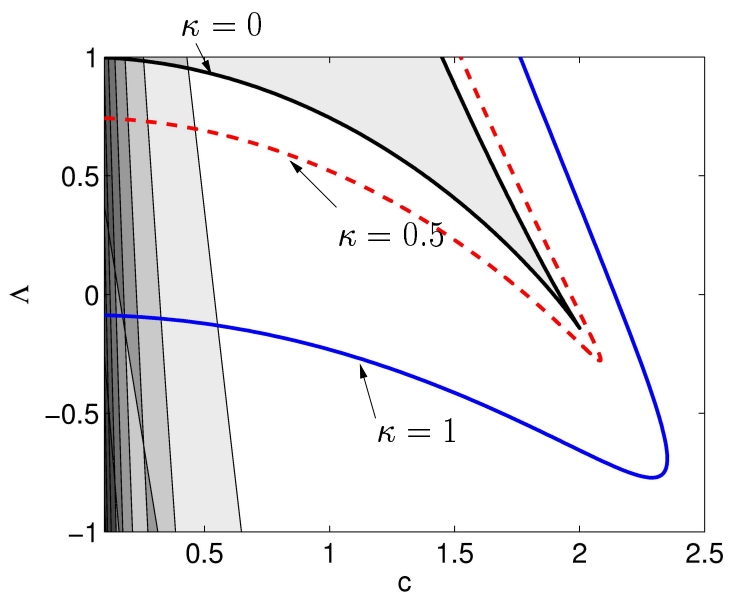

(b)

Figure 6: Spectral bands, in the $(\varepsilon, c)$ plane (a), and the $(c, \Lambda)$ plane (b). Overlaid in thick lines are the decay rate contours, (9), for the fundamental spectral band $\lambda \in(0, \pi)$ for fixed values of $\kappa . \kappa=0$ corresponds to the edges of the spectral bands, shown in thick black line. $\kappa=0.25$, dash-dotted line. $\kappa=0.5$, dashed line. $\kappa=1$, solid line.

\section{Numerical Methods}

Taking as our starting point the travelling wave form of the discrete nonlinear Schrödinger equation with a photorefractive nonlinearity (3), a pseudo-spectral substitution is used to transform (3) into a system of algebraic equations. To do this we follow a similar methodology as presented in [2], using a finite Fourier series expansion to approximate the discrete soliton, $\psi(z)$, on a long finite interval [-L/2, L/2]. Our choice of expansion terms is governed by out earlier choice that $\psi(0)$ should be real and the realisation that equation (5) is invariant under the reversing symmetry $z \rightarrow-z, \psi \rightarrow \psi^{*}$. We are seeking fundamental "single hump" solitons therefore we shall look for solutions that are invariant under this reversibility, which leads 
us to choosing an expansion which has even real functions and odd imaginary functions:

$$
\begin{aligned}
\psi(z) & =\sum_{j=1}^{N} a_{j} \cos \left(\omega_{j} z\right)+i b_{j} \sin \left(\omega_{j} z\right), \\
\omega_{j} & =\frac{\pi j}{L},
\end{aligned}
$$

where $a_{j}, b_{j} \in \mathrm{R}$ are the coefficients of the Fourier series. Substituting this into (3) gives a system of $2 N$ algebraic equations for the unknown coefficients $a_{j}, b_{j}$ :

$$
\begin{aligned}
\operatorname{Re}(F(z))= & \sum_{j=1}^{N}-c b_{j} \omega_{j} \cos \left(\omega_{j} z\right)+(2 \varepsilon-\Lambda) a_{j} \cos \left(\omega_{j} z\right)-\varepsilon\left(a_{j} \cos \left(\omega_{j}[z+1]\right) \cos (k)\right. \\
& \left.-b_{j} \sin \left(\omega_{j}[z+1]\right) \sin (k)+a_{j} \cos \left(\omega_{j}[z-1]\right) \cos (k)-b_{j} \sin \left(\omega_{j}[z-1]\right) \sin (k)\right) \\
& +\frac{1}{1+\left|\left(\sum_{m=1}^{N} a_{m} \cos \left(\omega_{m} z\right)+i b_{m} \sin \left(\omega_{m} z\right)\right)\right|^{2}} a_{j} \cos \left(\omega_{j} z\right)=0, \\
\operatorname{Im}(F(z))= & \sum_{j=1}^{N}-c a_{j} \omega_{j} \sin \left(\omega_{j} z\right)+(2 \varepsilon-\Lambda) b_{j} \sin \left(\omega_{j} z\right)+\varepsilon\left(b_{j} \sin \left(\omega_{j}[z+1]\right) \cos (k)\right. \\
& \left.+a_{j} \cos \left(\omega_{j}[z+1]\right) \sin (k)+b_{j} \sin \left(\omega_{j}[z-1]\right) \cos (k)-a_{j} \cos \left(\omega_{j}[z-1]\right) \sin (k)\right) \\
& +\frac{1}{1+\left|\left(\sum_{m=1}^{N} a_{m} \cos \left(\omega_{m} z\right)+i b_{m} \sin \left(\omega_{m} z\right)\right)\right|^{2}} b_{j} \sin \left(\omega_{j} z\right)=0 .
\end{aligned}
$$

These equations are posed on the collocation points $z_{i}$ where:

$$
z_{i}=\frac{L i}{2(N+1)} .
$$

We now have a large system of nonlinear algebraic equations which are solved numerically for a fixed set of parameter values $(c, \varepsilon, \Lambda)$ using the Powell hybrid method [35], as implemented using the Minpack routines [36], with an error tolerance of $10^{-13}$. The Powell hybrid method uses a combination of steepest descent and Newton directions, with a weighting of $\beta_{k}$ so that $0<\beta_{k}<1$ can be selected to vary which direction is used,

$$
x_{k+1}=x_{k}+\beta_{k} d_{k}+\left(1-\beta_{k}\right) h_{k},
$$

where $h_{k}$ is the Newton direction and $d_{k}$ is the steepest descent. The parameter $\beta_{k}$ is related to the maximum step size which is updated using a an algorithm that gives a bias toward steepest decent when convergence is slow and a bias toward the Newton direction if convergence is fast. The Powell hybrid method has been chosen as it is globally convergent. Using the Powell hybrid algorithm gives a solution of (12) and (13) for a given initial guess of coefficients $a_{j}, b_{j}$. This solution can then be converted back in terms of $\psi(z)$ allowing us to study the form of the solution obtained by the fixed point method. Any solution that is found using this method can then be numerically continued using AUTO [8], with error tolerance $10^{-10}$, allowing us to investigate the effect of varying parameters on solution shape and especially tail amplitude.

The solutions found using the Powell Hybrid method will generally be weakly non-local solitary waves [4] or quasi-solitons [23] and exhibit non-zero oscillatory tails; see, for example, Figure 7. To find solutions with non oscillatory tails we need to add an extra tail condition, For this condition we use a signed measure of the amplitude of the tail and seek to find zeros of this function. There are a number of candidates for this 


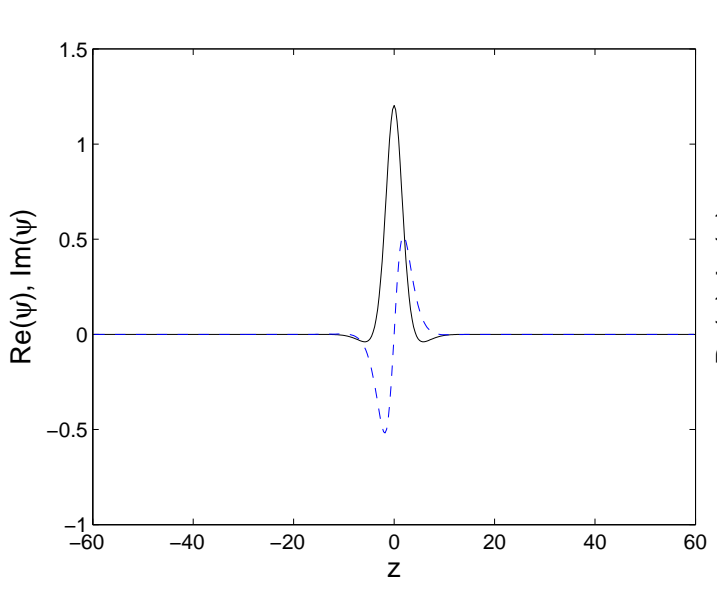

(a)

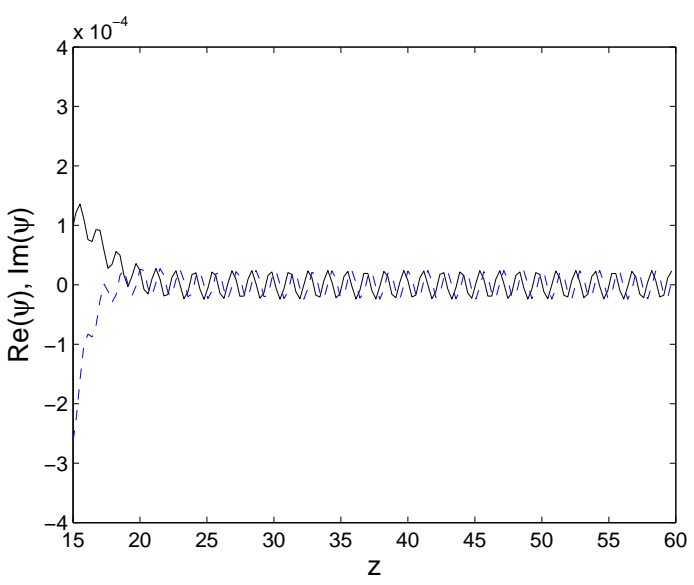

(b)

Figure 7: Results obtained using the Powell hybrid method with $\varepsilon=1, c=0.7, \Lambda=0.5, L=120$. Solid line indicates $\operatorname{Re}(\psi)$, dashed line shows $\operatorname{Im}(\psi)$. (a) Shape of full solution. (b) Magnification of the tail exhibiting oscillations.

tail function such as those used in [28] and [2], which measure the amplitude of the imaginary part of $\psi$ or the gradient of the real part of $\psi$ in the tail of the solution respectively. It would also be possible to use the magnitude of $\psi$ in the tail however this would not give a signed measure of the tail amplitude and would therefore be less reliable as localised solutions would correspond to zeros of a quadratic function instead of as regular zeros of the tail function. We will choose to use the measure used in [28], namely

$$
\Delta=\operatorname{Im}\left(\psi\left(\frac{L}{2}\right)\right)
$$

Eq. (15) is a measure of the amplitude of the imaginary part of the tail of a solution of period L. We typically consider a travelling solitary wave solution to (3) as being made up of two parts, an exponentially localised core, $\psi_{\text {core }}$, and a non-vanishing oscillatory background $\psi_{\text {tail }}$ :

$$
\psi(z)=\psi_{\text {core }}(z)+\psi_{\text {tail }}(z)
$$

At a sufficient distance from the centre of the soliton $\psi_{\text {core }}$ will be zero due to its exponential localisation and therefore $\Delta$ is purely a measure of $\psi_{\text {tail }}$. Because of the way $\psi(z)$ has been approximated in Eq. (10), we know that the real part of $\psi(z)$ is odd around $z=L / 2$ and the imaginary part is even therefore (15) is a pure measure of the amplitude of the tail if $L$ is large enough.

If a zero of $\Delta$ is found then this implies that the tail of the travelling wave will be everywhere zero, $\left(\psi_{\text {tail }}(z)=0, \forall z\right)$. Therefore altering the period, $L$, of the solution would not change the solution, however if a value of $L$ is chosen too small then at the point the tail condition is applied, $\psi_{\text {core }} \neq 0$ and so $\Delta$ will not accurately reflect the amplitude of the tail. The accuracy of the solutions found with vanishing tail amplitude can be seen in Figure 9 where it is observed that the tail is zero up to the numerical accuracy used (in this case $O\left(10^{-10}\right)$ ). If a zero of $\Delta$, is found it can be numerically continued in $\mathrm{L}$ and another problem parameter. If $\mathrm{L}$ is chosen appropriately large so that $\psi_{\text {core }}(L / 2)=0$, then increasing it further should have no effect on the parameter values of the point $\Delta=0$, whilst if $L$ is too small and $\psi_{\text {core }}(L / 2) \neq 0$ then we would expect the parameter value at which $\Delta=0$ is found to vary with $\mathrm{L}$. As can be seen from Figure 8 , choosing $L \lesssim 55$ for branches I \& II and $L \lesssim 70$ for branch III indicates that the tail is being measured too close to the core and so (15) is not a true measure of the amplitude of the tail as there are still some influences from the core of the solitary wave. For very large values of $\mathrm{L}$ another problem can be seen to 


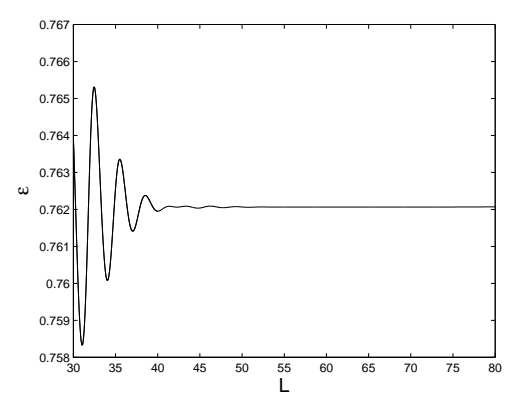

(a)

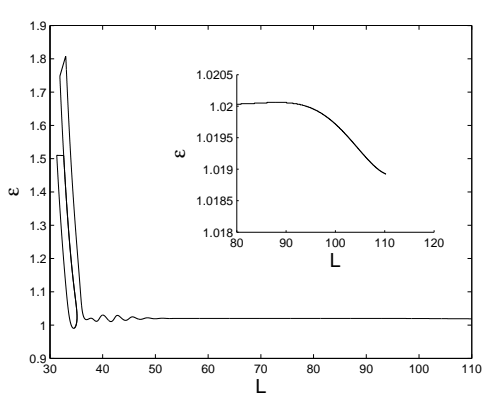

(b)

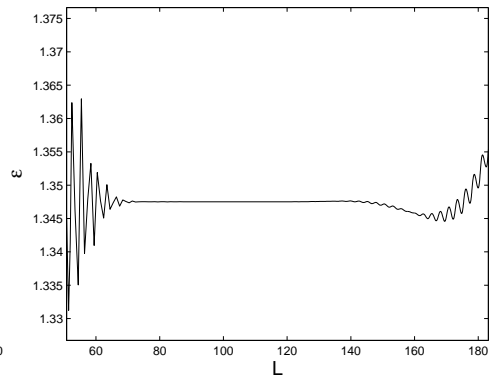

(c)

Figure 8: Continuation of three zeros of $\Delta$ in $L$ and $\varepsilon$ with $c=0.7, \Lambda=0.5$ with $N=200$ (a) \& (b) and $N=300$ (c). The inset shows the variation in $\varepsilon$ for large $\Delta$ due to the value of $\mathrm{N}$ being taken too small. (a) belongs to Branch I, (b) to Branch II, and (c) to Branch III, in the notation used below.

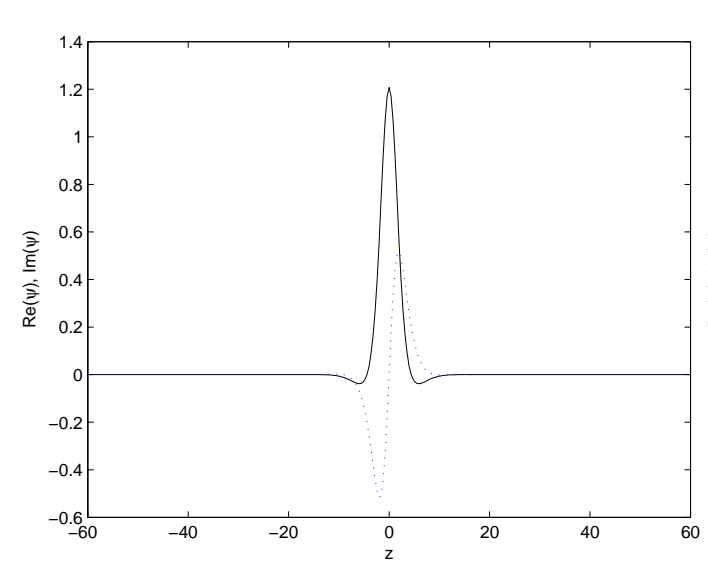

(a)

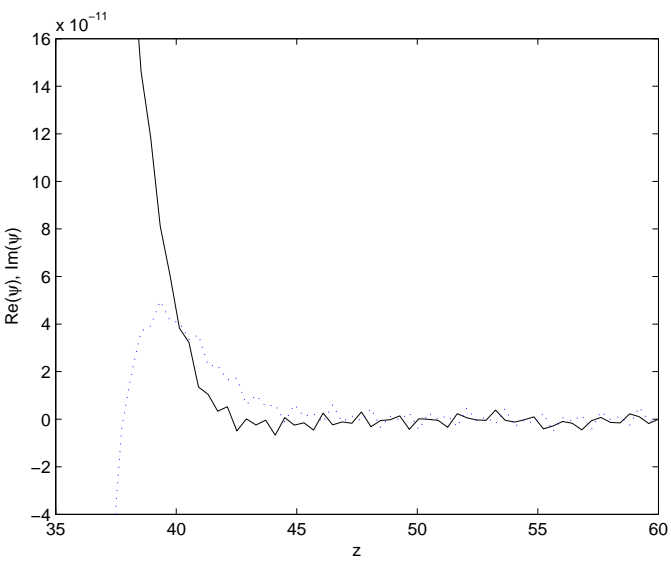

(b)

Figure 9: Travelling solitary wave of Branch II with $\varepsilon=1.020, c=0.7037, \Lambda=0.5, L=120, \Delta=0$. The solid line indicates $\operatorname{Re}(\psi)$, while the dashed line shows $\operatorname{Im}(\psi)$. (a) Shape of full solution. (b) Magnification of the tail showing it is zero to the numerical accuracy of the solver $\left(10^{-10}\right)$.

manifest itself in Figure 8 panel (b) inset and more clearly in panel (c); that is if $\mathrm{L}$ is increased beyond a certain point, then the collocation points $z_{i}$ used will be quite far apart and will not accurately represent a soliton solution. Hence the parameter values at which $\Delta=0$ is found will not represent a true solitary wave solution. This problem can, in principle, be overcome by increasing the number of collocation points $N$. However, we note that the optimal number of collocation points is difficult to predict and varies with solution parameters.

\section{Continuation results}

We also note that for large values of $c$ and $\varepsilon$, the solution branches become more difficult to calculate as larger values of $L$ and $N$ have to be taken to ensure that $\Delta$ is still a true measure of the tail amplitude. As $\varepsilon$ is increased the solution curves in Figure 10 appear more like a square well in shape and it therefore tends to become more difficult to isolate a true zero of $\Delta$ from a nearby nonzero but still very small value of $\Delta$. Hence, the computation of the exact parameter value at which any subsequent solitary wave branch would 


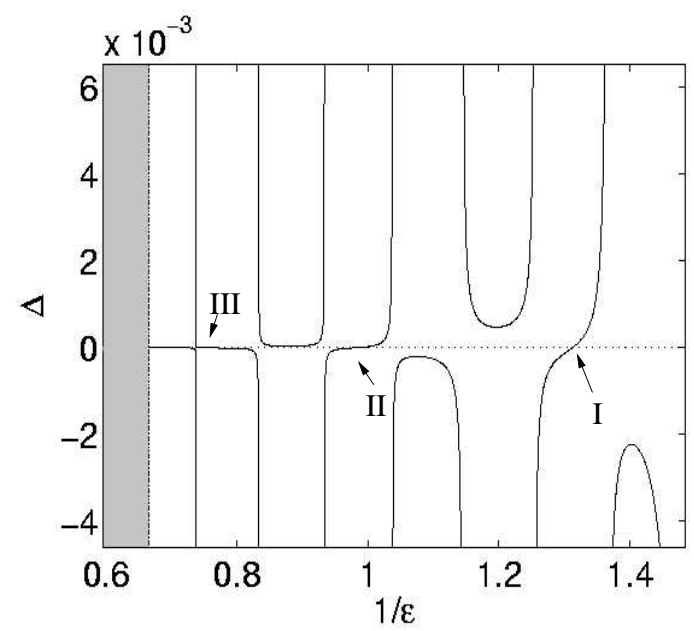

Figure 10: Continuation of weakly non-local solitons for various values of $1 / \varepsilon$ showing three zeros in $\Delta$ for $c=0.7, \Lambda=$ $0.5, L=60$. Zeros of $\Delta$ at $\varepsilon \approx 0.76,1.02,1.36$. The shaded region represents the spectral band where any embedded solitons would be of co-dimension 2 .

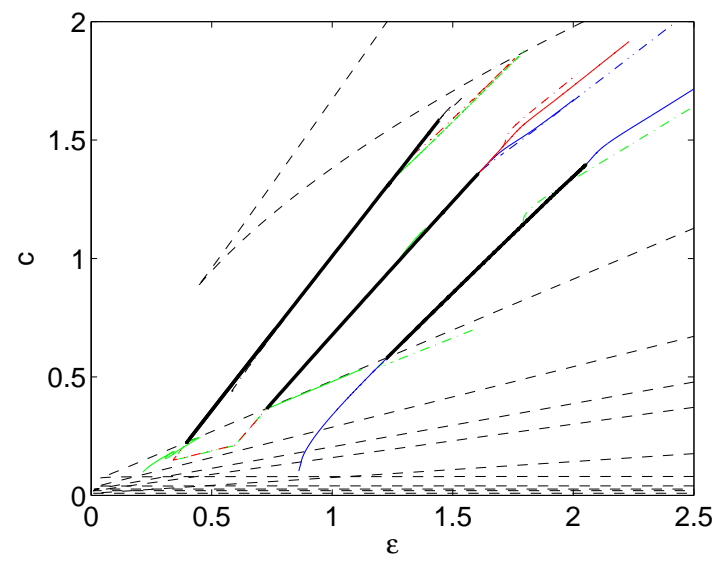

(a)
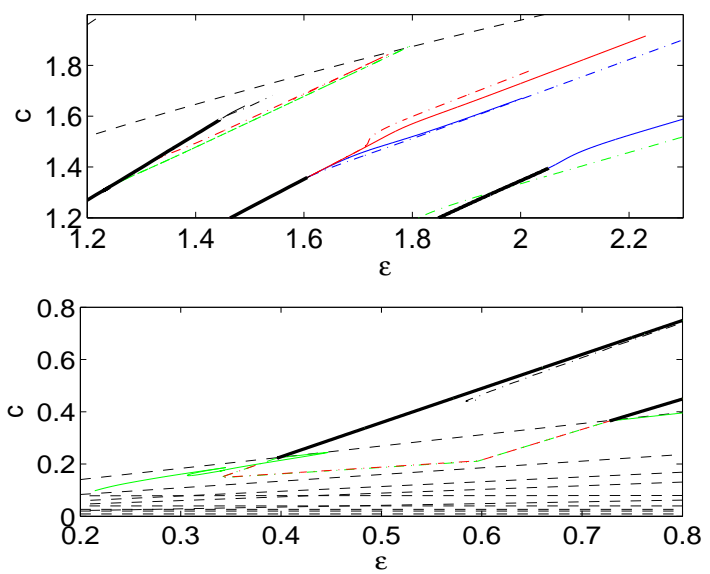

(b)

Figure 11: Continuation of the 3 zeros of $\Delta$ shown in Figure 10, varying $\varepsilon$ and $c$ with $\Lambda=0.5$. The continuation curves diverge for different values of $\mathrm{L}$ near the spectral bands as explained in the text. Solid black lines indicate the solution branches shown in Figure 13, black dashed-dotted lines are for $L=280$, solid red lines - $L=130$, red dashed-dotted lines - $L=120$, blue solid lines - $L=110$, blue dashed-dotted lines - $L=90$, green dashed-dotted lines - $L=80$, green solid lines $-L=60$. (b) Detail of the upper part of branch II, top panel, and lower part of branch I, bottom panel. clearly showing divergence for differing values of L.

would have zero tail amplitude becomes increasingly cumbersome. The solution branches shown are easily repeatable for differing values of $L$, Figure 11 shows the effects of varying $\mathrm{L}$ in the continuation of solitary wave branch. Note, in particular, the differing behaviour as a branch approaches the lower spectral bands and the 'kinks' in the upper parts of the solution curves as they approach the upper spectral band; this effect is due to the value of $L$ being too small and therefore $\psi_{\text {core }}$ being non-zero in the tail.

Once a weakly non-local soliton is found using the Powell-Hybrid method, the solution is continued in $(\Delta, W)$, where $W$ is any chosen parameter. If a solution which passes through $\Delta=0$ is identified, in a way similar to what is shown in Fig. 12, then $\Delta$ can be fixed there and the solution can be continued in any two parameters from $(\varepsilon, c, \Lambda, L)$. Using this method a number of different zeros of $\Delta$ are found as shown in 


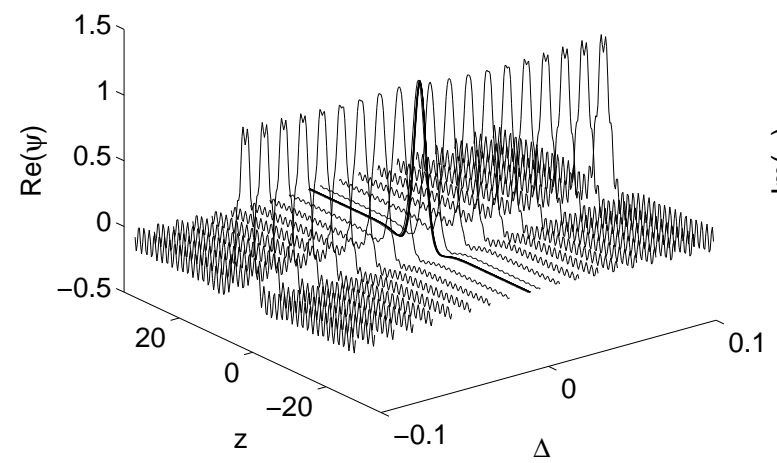

(a)

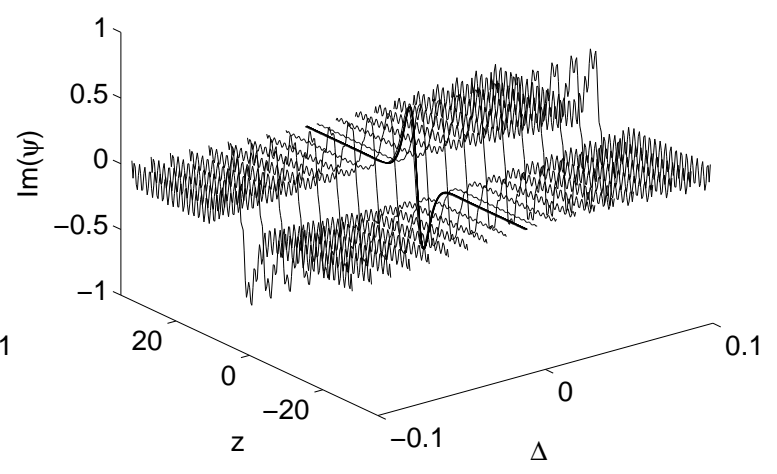

(b)

Figure 12: Continuation of branch with second zero of $\Delta$ at $\varepsilon \approx 1.02$. for $c=0.7, \Lambda=0.5, L=60$. (a) $\operatorname{Re}(\psi)$, (b) $\operatorname{Im}(\psi)$.

Figure 10. Notice the difference between the ' $U$ ' and ' $n$ ' shaped curves which do not pass through $\Delta=0$ and the ' $S$ ' shaped curves which include the zeros of $\Delta$. It can be seen that these curves appear periodic in $1 / \varepsilon$ and are of similar form to those found in previous works [5] [23]. Finding expressions for these curves is a regular asymptotic problem involving the computation of only the first few terms of an asymptotic expansion and will be discussed in future work. However obtaining parameter values where the curves pass through $\Delta=0$ is a much more difficult, beyond all orders asymptotic problem due to the amplitude of the tails becoming exponentially small as $\varepsilon$ approaches $\Delta(\varepsilon)=0$, [31].

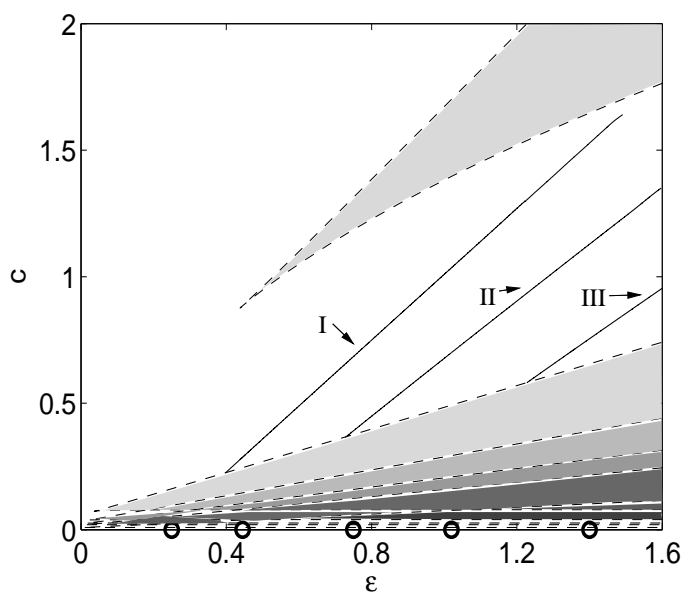

Figure 13: Continuation of the 3 zeros of $\Delta$ shown in Figure 10, varying $\varepsilon$ and $c$ with $\Lambda=0.5$. Circles on the $c=0$ axis indicate the transparent points of (1).

By continuing the three zeros of $\Delta$ in Figure 10 in the parameters $(c, \varepsilon)$ with $\Delta=0$ the effects of the multi-phonon bands, Figure 3, on the solitons can be investigated. Continuation of the three zeros of $\Delta$ found in Figure 10 are shown in Figure 13. The continuation curves for a particular zero of $\Delta$ above the multiphonon bands for differing values of $L$ are the same, but they differ when they enter the lower phonon bands 
or approach the upper phonon band, either terminating at the edge or losing their exponential localisation when entering the band. This behaviour indicates that the true solitary wave continuation branches cannot be continued inside the multi-phonon band and, in fact, terminate at the boundary of the spectral bands where an extra pair of purely imaginary eigenvalues appear. Profiles along branch I are shown in Figure 14 where the broadening of the soliton for large $c$ as it approaches the upper phonon band can easily be seen. Branches II and III have qualitatively similar profiles. It is interesting to note that although the solution branches in Figure 13 cannot be continued into the spectral bands and down to the line $c=0$, it appears that they do in fact originate from the first three transparent points of (1). It may therefore be expected to be possible to find further branches of $\Delta=0$ for larger values of $\varepsilon$ and $c$.

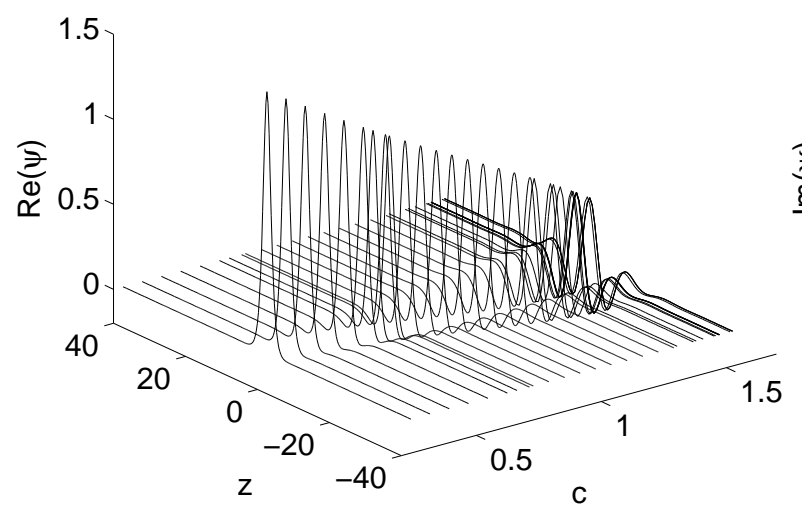

(a)



(b)

Figure 14: Continuation of the first branch of solutions with varying $\varepsilon, c$ and $\Lambda=0.5$ (a) $\operatorname{Re}(\psi(z))$, (b) $\operatorname{Im}(\psi(z))$. The lower portion of the branch is computed with $L=60$, if the solution is continued inside the multi-phonon band it becomes delocalized. As $c$ is incresed larger values of $L=80$ and 280 are used to capture the solution as the soliton core expands. Near the upper phonon band the soliton branch terminates. Solutions have been truncated in $z$ for ease of viewing.

The parameter $\varepsilon$ is fixed by the governing equation (1), whilst $\Lambda$ and $c$ are dependent upon the solution ansatz, equation (2) therefore to investigate how the solution profiles may vary for a given lattice it makes sense to fix the parameter $\varepsilon$ at some value and then continue the soliton branches in the solution parameters $c$ and $\Lambda$, as is shown in Figure 15. Setting $\varepsilon=1$ we can use the solutions for branches I \& II already used in this section as a starting point, a branch III solution for $\varepsilon=1$ can be found by continuing the solution used in this section in the parameters $\varepsilon, \Lambda$ until $\varepsilon=1$. Using this method a number of other solution branches can also be found for smaller values of $\Lambda$. As $\Lambda$ is decreased and the gap between each successive branch decreases. It is expected that many more branches than shown would exist if $\Lambda$ is decreased, although as the branches appear closer together it becomes difficult to distinguish between them. None of the extra branches found here (IV - VII) intersect the line $\Lambda=0.5$ and so it would not be possible to see these branches for $\varepsilon=1$ in Figure 13, however in a similar fashion to branch III, these extra branches could appear for larger values of $\varepsilon$ originating from higher transparent points.

As can be seen in Figure 15, the continuation branches again terminate at the spectral bands for small $c$, as they should, whilst for large wavespeeds the branches seem to converge to a point for $(c, \Lambda) \approx(2,-1)$ although in this limit the amplitude of the solitary waves becomes very large and it is debatable whether 


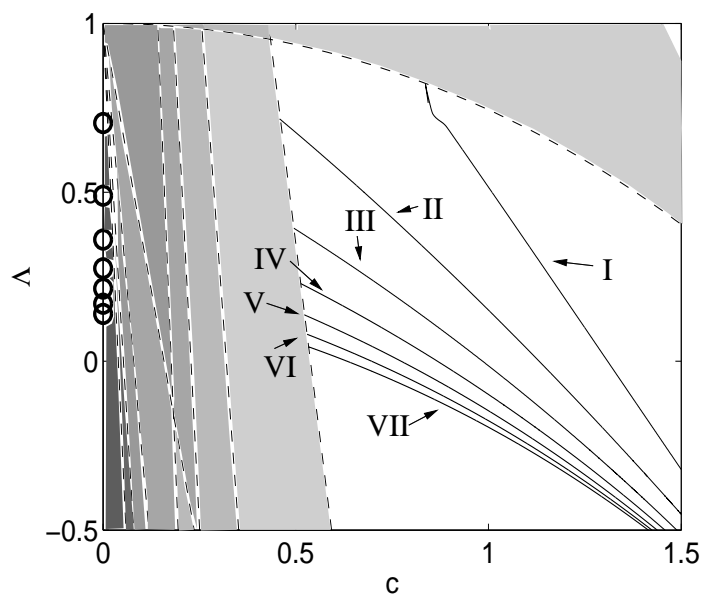

Figure 15: Continuation of the 3 solitary wave branches with varying $\Lambda$ and $c$ for fixed $\varepsilon=1$. Extra branches, labelled IV - VII are also found for smaller values of $\Lambda$. Branches appear to converge in the large wavespeed limit.

these represent valid solutions. The variation in amplitude of the solutions along the continuation curves for Figures 13 and 15 are shown in Figure 16. As $\Lambda$ is increased the wave amplitude decreases. The amplitude of branch I tends to zero in both cases (where for the $(\varepsilon, c)$ plane the first branch has been extended by using a solution for which $\psi_{\text {core }} \neq 0$ in the tail. However we would expect the same qualitative behaviour of the core amplitude if the solution was continued to the edge of the spectral band) as it approaches the fundamental spectral band due to the related eigenfunction of the fundamental band, Figure 6. See [32], [31] for analysis and results around this band. All other branches terminate at a spectral band with non-zero amplitude. Apart from this difference in amplitude there is little qualitative difference between the solutions on each branch, in particular all solutions are single humped.

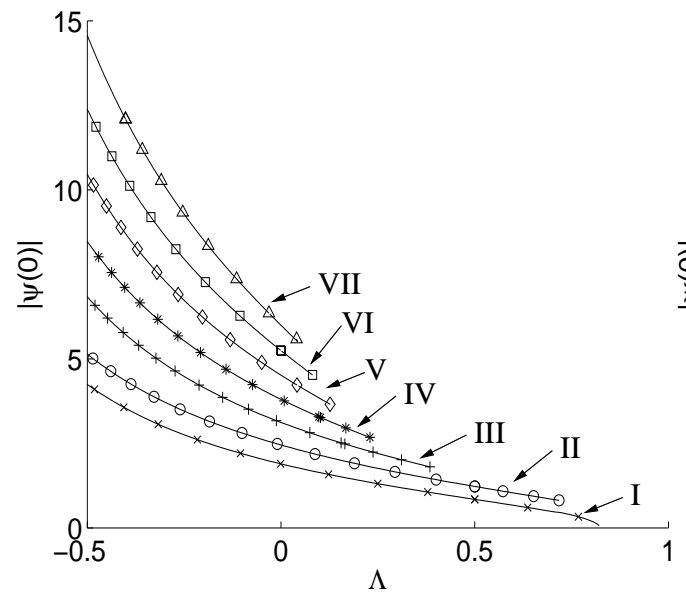

(a)

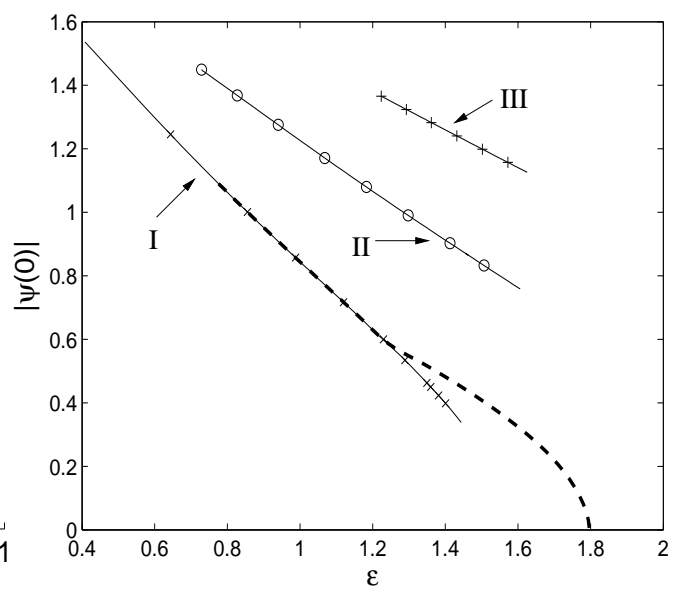

(b)

Figure 16: Amplitude of solutions, $|\psi(z=0)|$ along the continuation branches shown in (a) Figure 15 as $\Lambda$ varies and (b) from Figure 13 as $\varepsilon$ varies. Thick dashed line in panel (b) is from a solution on Branch I for which $\psi_{\text {core }} \neq 0$ in the tail hence $L$ is too small, however this gives a good indication that the soliton amplitude goes to zero as it intersects the upper phonon band.

To confirm that no branches have been missed in between the ones shown in Figure 15 (although it 
would be expected that there are more branches below the ones shown), the transparent points of the grandcanonical energy $G$ are again calculated. Taking a stationary solution computed from the solution at the anti-contimuum limit to the point $\varepsilon=1$ for a fixed value $\Lambda=0.5$ and continuing in $\Lambda$ yields Figure 1 (b). A number of transparent points are found, becoming denser as $\Lambda$ approaches zero. However, near $\Lambda=0$ and 1 the continuation breaks down. This break down is due to the amplitude of the stationary profiles being related to the value at the anti-continum limit of $\pm \sqrt{(1 / \Lambda)-1}$ so that as $\Lambda$ approaches zero the amplitude blow up and at $\Lambda=1$ the amplitude is zero, therefore this analysis is only valid in the region $\Lambda \in(0,1)$ and so the transparent points from which the first two branches orginate cannot be found using this method, although for a fixed $\Lambda$ and varied $\varepsilon$ they can be found as regular transparent points, see Figure 1(a).

\section{Direct Numerical Integration}

We can use the solutions obtained in the previous section (as stationary solutions in the travelling frame) as initial conditions in numerical simulations of equation (1). A $4^{\text {th }}$ order Runge-Kutta method as implemented by Matlab, with variable time step and absolute tolerance $10^{-10}$, is used to solve the initial value problem with periodic boundary conditions $u_{n+N}=u_{n}$. This method only requires data at the preceding time step so only the initial data (and the lattice parameter $\varepsilon$ ) need to be specified. On the other hand, $(c, \Lambda)$ are solution parameters and are not prescribed a priori but are intrinsic characteristics of each solution which would be expected to occupy a single point in $(c, \Lambda)$ space for a given $\varepsilon$. We wish to study the temporal evolution of the travelling solitary waves found in section 4 and their "robustness" (i.e., stability) under perturbations, as well as their interactions. A typical example of the evolution of a single soliton with $\varepsilon=1$ from branch II is shown in Figure 17. As can be inferred from the dynamics, the solution appear to be steadily and robustly propagating with a constant slope on the space-time diagrams (i.e., a constant speed), as anticipated. This has been repeated for solitons on branches I and III with similar results which have been excluded here for brevity.

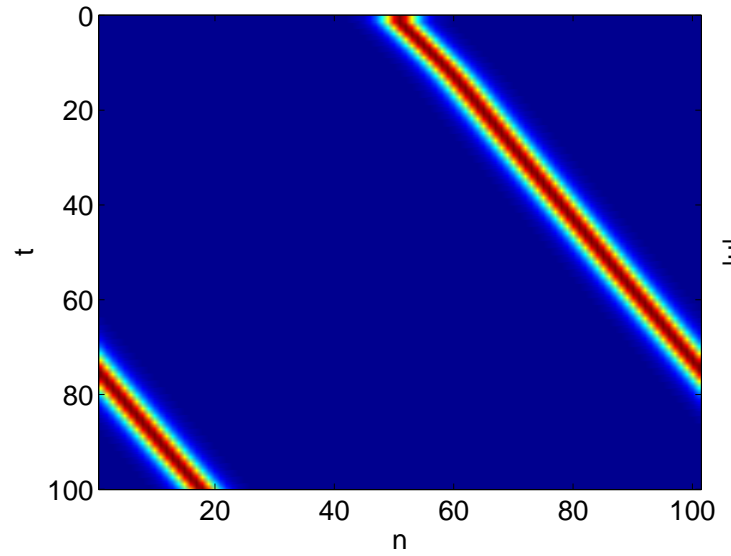

(a)

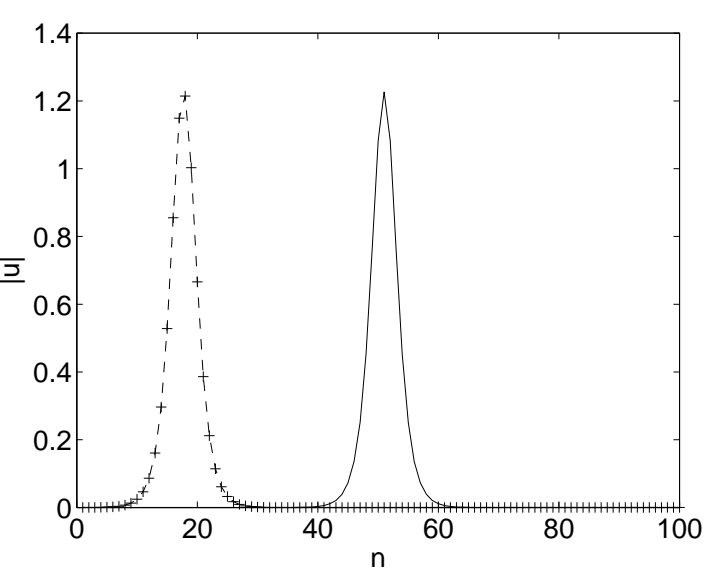

(b)

Figure 17: Numerical Integration of the solution of Branch II in Figure 13 with $\varepsilon=1, c=0.67725$ with $\Lambda=0.5, N=100$. (a) Motion of the solution through the lattice for 100 time steps (shown is a space-time contour plot of the modulus of the solution), (b) Initial and final profiles of the wave after 100 time steps; the final lattice node points are indicated with a + . 


\subsection{Stability of Travelling Waves Under Perturbation}

The direct integration discussed above can be used to investigate the stability of the solutions obtained by numerical continuation. It has been shown that embedded solitons can be considered as semi-stable objects [45], that is if they are perturbed by adding energy to them then the soliton will relax back to its original state by shedding off the excess energy as radiation in the lattice whilst if they are perturbed by reducing their energy then the soliton will become delocalised and radiate all energy away accross the lattice. However here we find that our solutions appear in fact to be stable to amplitude perturbations, Figures 18 - 20.



(a)

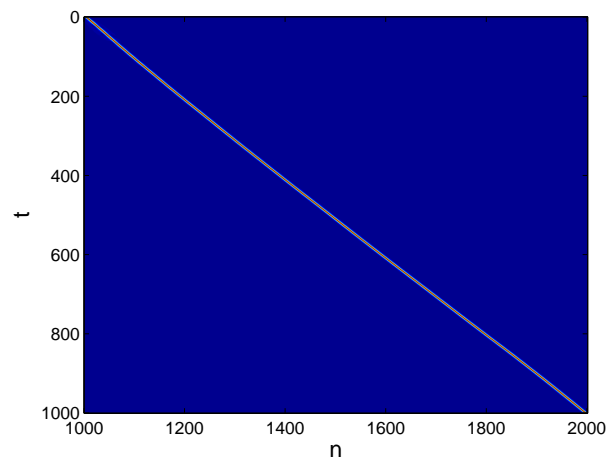

(c)
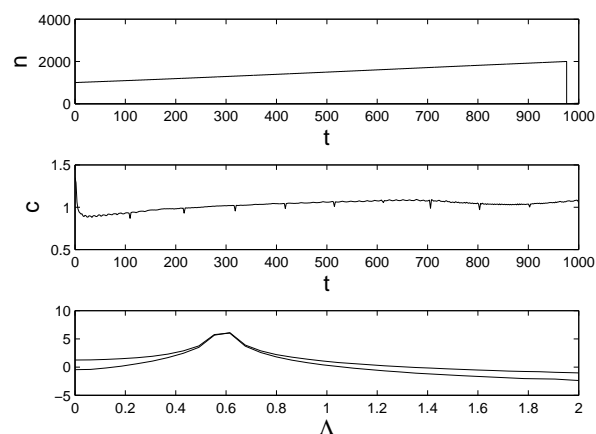

(b)
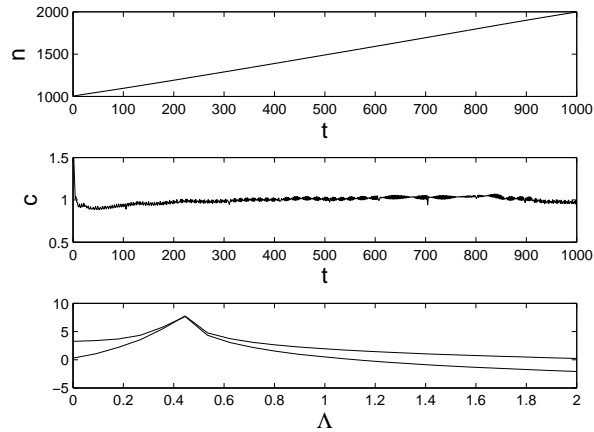

(d)

Figure 18: Direct integration of a perturbed solution from Branch I in Figure 13 with $\varepsilon=1$ and $c=1.00926, \Lambda=0.5, N=$ 2000. (a) Motion of the solution with $\alpha=0.8$ through the lattice for 1000 time steps, (b) the top panel shows the position of the soliton at time $t$, the middle panel shows the velocity based upon numerical differentiation, and the lower panel shows the log of the power spectrum indicating a shift in frequency from the unperturbed value $\Lambda=0.5$. (c) and (d) show the same as (a) and (b) but with $\alpha=1.2$

These different types of perturbations can be simulated by direct integration using initial profiles that represent different fractions of the solution profiles obtained in the previous sections, $v_{n}(0)=\alpha u_{n}(0)$ where $u_{n}(0)$ is an initial solution taken from one of the branches in Figure 13 and $\alpha$ is a constant used to vary the initial profile ( $\alpha=1$ would correspond the unperturbed solution). The results of these simulations for $\alpha \neq 1$ are shown for a branch I solution in Figure 18. Figure 19 shows the variation in position, wavespeed and frequency of perturbed solutions on branches II and III where qualitatively similar results are found to perturbed solutions on branch I. When the initial profile is perturbed instead of either relaxing back to the unperturbed solution if extra energy is added or becoming delocalised if energy is taken away, as might be expected if the solutions were in fact semi-stable. Instead it appears that another travelling soliton solution is selected but with different frequency $\Lambda$ and wavespeed $c$. The peak in the frequency spectrum can be seen to 

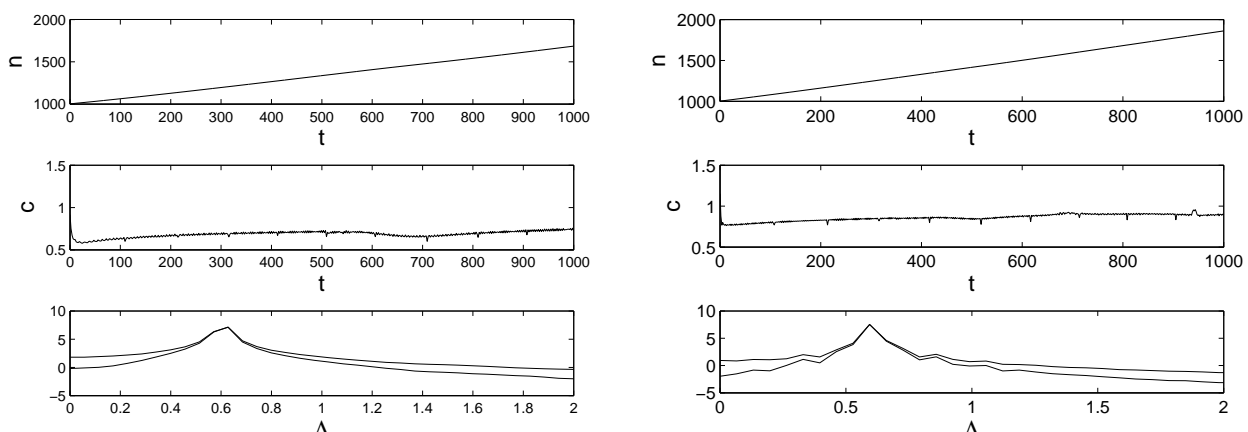

(a)

(b)
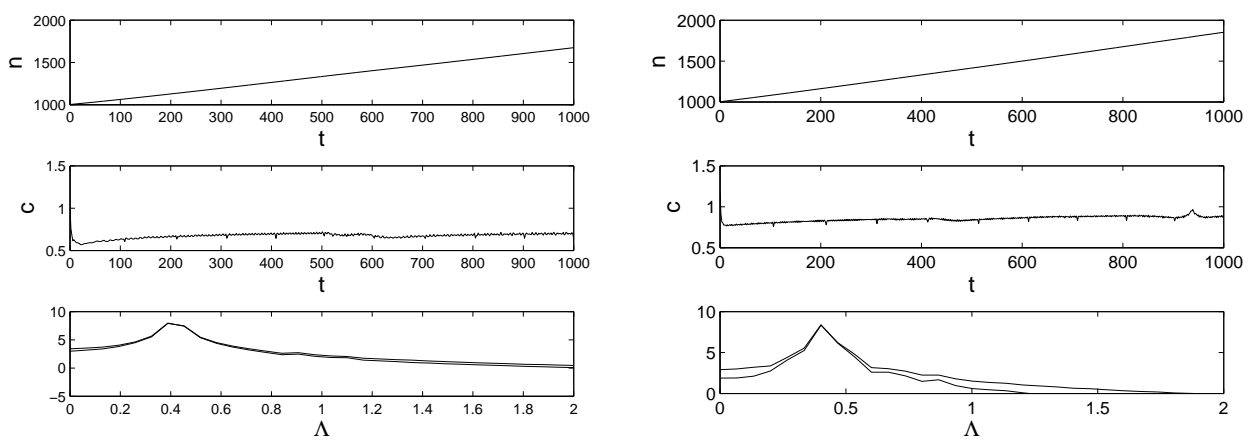

(c)

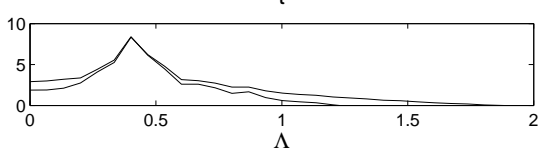

(d)

Figure 19: Variation in position, wavespeed, $c$ and frequency $\Lambda$ for perturbed solutions on branch II with (a) $\alpha=0.8$ and (b) $\alpha=1.2$. Branch III with (c) $\alpha=0.8$ and (d) $\alpha=1.2$. Panels are the same as for Figure 18 (b) and (d).

move, Figure 18 panels (a) and (c) and Figure 19, from the unperturbed value of $\Lambda=0.5$ and the wavespeed $c$ also exihibits a small variation although this is difficult to detect. Figures 20-21 show a clearer view of the frequency and amplutide variation of the perturbed solutions. If $\alpha>1(<1)$ then $\Lambda, c$ decreases (increases). During an initial transition period where a the soliton settles into the "new" solution, a certain amount of radiation is shed and the velocity is distinctly higher than that of the unperturbed soliton, see panels (b) \& (d) in Figure 18 . After this transient, the soliton settles into the new solution, although as compared with the unperturbed solution the amplitude is often oscillatory, see Figure 20, and the new wavespeed is not constant but often slightly oscillatory with periodic "dips". The rapid oscillations that appear in the values of $\left|u_{n_{\text {Max }}}\right|$, where $n_{\text {Max }}$ is the lattice site with largest the value of $|u|$, in Figure 20 is due to $\operatorname{Re}\left(u_{n}\right)$ and $\operatorname{Im}\left(u_{n}\right)$ not being exactly out of phase so that $\left|u_{n}\right|$ appears oscillatory. The change in the soliton frequency due to variations of $\alpha$ is shown in Figure 21 for Branches I \& II, as can be seen the new soliton profiles stay very close to the unperturbed solution branch even for a large perturbation, $\alpha=0.5,1.4$.

\subsection{Orbital Stability of Travelling Waves}

The above results already illustrate the stability of this branch of travelling solutions. However, to corroborate these findings we have also used linearization techniques in the form of linear stability analysis. we introduce a small perturbation $\xi_{n}$ to a given solution $u_{n 0}$ (of branches I-III) of Eq. (1) according to 

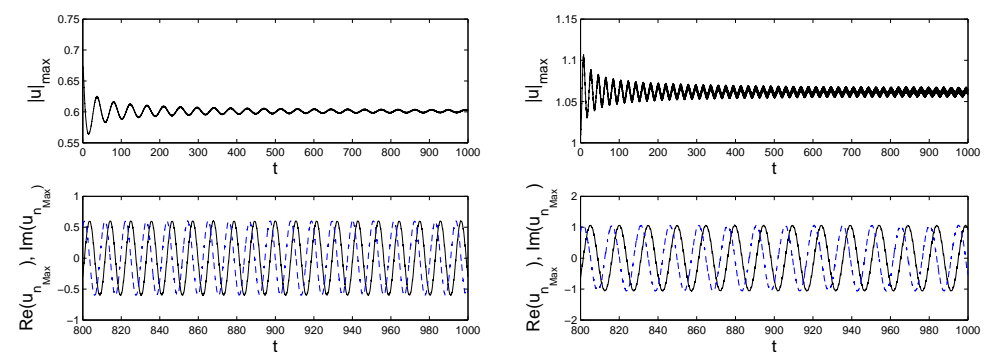

(a)

(b)

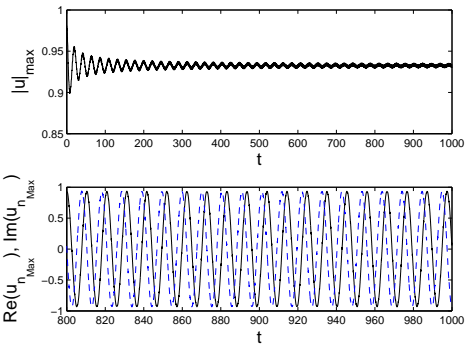

(c)

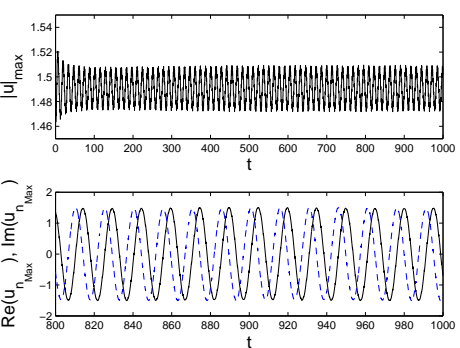

(d)

Figure 20: Profiles of perturbed embedded solitons, (a) Branch I soliton with $\alpha=0.8$, (b) Branch I soliton with $\alpha=1.2$, (c) Branch II soliton with $\alpha=0.8$, (d) Branch II soliton with $\alpha=1.2$. Top panels show $|u|$ for soliton and bottom panels. $\operatorname{Re}(u)$ solid line, $\operatorname{Im}(u)$ - dashed line.

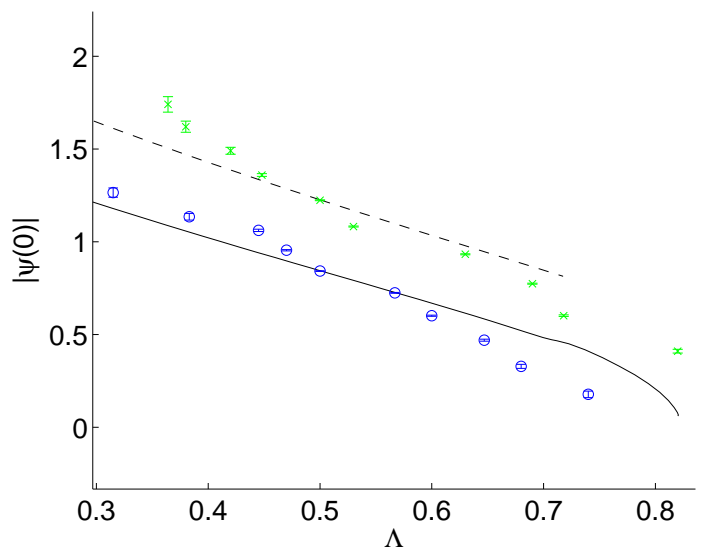

Figure 21: Change in frequency, $\Lambda$, of a soliton due to perturbation of the initial profile with $\Lambda=0.5, \alpha \in(0.5,1.4)$. Branch I, solid line indicates unperturbed solution amplitudes, with perturbed solutions shown by circles. Branch II, dashed line indicates unperturbed solution amplitudes, perturbed solutions indicated by crosses. Error bars indicate the variation in amplitude.

$u_{n}=u_{n 0}+\xi_{n}$. Then, the equation satisfied to leading order by $\xi_{n}$ is:

$$
i \dot{\xi}_{n}-\frac{\beta}{1+\left|u_{n 0}\right|^{2}} \xi_{n}+\frac{\beta}{\left(1+\left|u_{n 0}\right|^{2}\right)^{2}}\left(\left|u_{n 0}\right|^{2} \xi_{n}+u_{n 0}^{2} \xi_{n}^{*}\right)+\epsilon \Delta \xi_{n}=0
$$

In order to examine the orbital stability analysis of the relevant solution, a Floquet analysis can be performed if there exist $T \in \mathbb{R}$ and $q \in \mathbb{Z}$ so that the map

$$
u_{n}(0) \rightarrow u_{n+q}(T)
$$



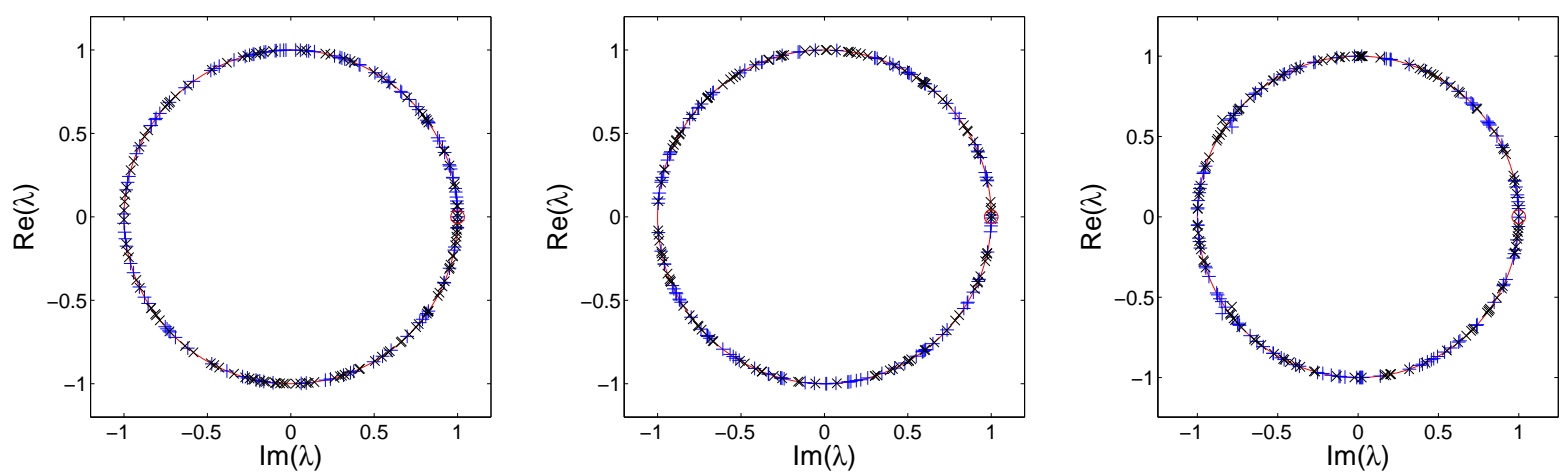

Figure 22: Floquet eigenvalues in the unit circle. Solutions corresponds, from left to right, to the 1st, 2nd and 3rd branches. In all cases, $q=8, p=1$. The Krein signature (see e.g., [19], [3]) is indicated by the following signs: Positive $(+)$, negative $(\times)$ and zero $\circ$

has a fixed point $[16,17]$. Then, the stability properties are given by the spectrum of the Floquet operator $\mathcal{F}$ (whose matrix representation is the monodromy) defined as:

$$
\left(\begin{array}{c}
\left\{\operatorname{Re}\left(\xi_{n+q}(T)\right)\right\} \\
\left\{\operatorname{Im}\left(\xi_{n+q}(T)\right)\right\}
\end{array}\right)=\mathcal{F}\left(\begin{array}{c}
\left\{\operatorname{Re}\left(\xi_{n}(0)\right)\right\} \\
\left\{\operatorname{Im}\left(\xi_{n}(0)\right)\right\}
\end{array}\right)
$$

The Floquet operator is symplectic, which implies that there is always a pair of degenerate monodromy eigenvalues (corresponding to the phase and growth modes [19], [3]) at 1. If the moving soliton is stable, all the eigenvalues must lie on the unit circle.

Given the spatial and temporal dependence of the solution, we expect the periodicity condition of the map (17) is fulfilled as long as the profile "recurrence" period $T$ is connected to the solution frequency through

$$
T=\frac{q}{c} \quad \frac{2 \pi c}{\Lambda}=\frac{q}{p}, p \in \mathbb{Z}
$$

This commensurability condition implies the absence of a continuous parameter set for which an orbital stability analysis can be performed. Instead, it is possible to examine the results of Floquet theory for isolated points along the branches I-III, as is shown in Fig. 22.

We have performed the stability analysis for a set of commensurate solutions of each branch, finding that the modulus of every eigenvalue is 1 . In consequence, we observe that the relevant examined solutions are orbitally stable ones. The values of $p: q$ chosen in each set of commensurate solutions (for which we have established the above notion of stability) are the following:

- 1st Branch. 15:4, 120:31, 4:1, 120:29, 30:7, 40:9, 60:13,24:5, 5:1, 120:23, 60:11, 40:7, 6:1, 120:19, $20: 3,120: 17,15: 2,8: 1,60: 7,120: 13,10: 1,120: 11,12: 1,40: 3,15: 1,120: 7$.

- 2nd Branch. 80:17, 5:1, 16:3, 40:7, 80:13, 20:3, 80:11, 8:1, 80:9, 10:1, 80:7.

- 3rd Branch. 15:2, 8:1, 60:7, 120:13, 10:1.

\subsection{Collisional Interactions Between Travelling Waves}

Lastly, we have tested the robustness of the travelling wave solutions under both symmetric (i.e., identical solutions moving in opposite directions) and non-symmetric (i.e., non-identical solutions moving in opposite directions or even solutions moving with different speeds in the same direction) conditions. 



Figure 23: Dependence of the restitution coefficient for symmetric collisions with respect to the frequency $\Lambda$ for branches I, II and III (from left to right). Circles correspond to reflection / refraction and triangles to breather creation.
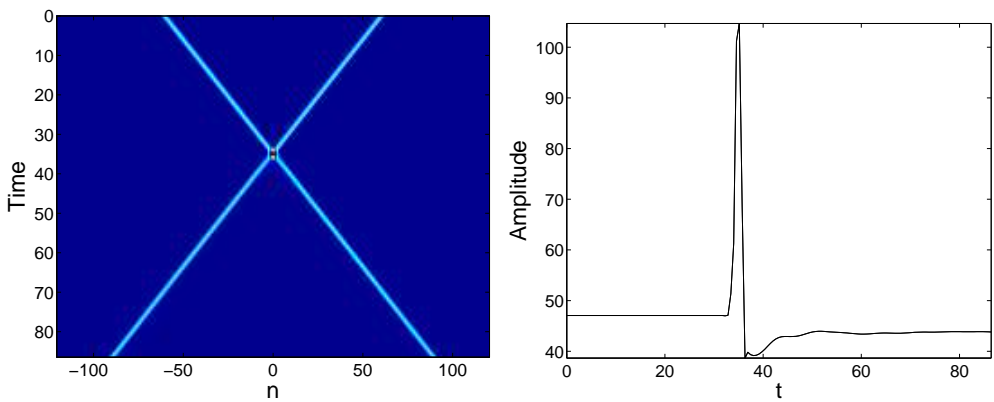

Figure 24: Collision of two solitary waves belonging to branch I with initial velocity $c=1.7351$ and $\Lambda=-0.7121$. They are refracted / reflected after the collision. (Left) Space-time contour plot of the solution density. (Right) Time dependence of the amplitude of the moving wave.

To this end, we have used in our collisional numerical experiments solutions of branches I, II and III in Figure 15 with fixed $\varepsilon=1$ and variable $\Lambda$ and $c$. As a quantitative diagnostic of the elasticity of the collision we have used the restitution coefficient, defined as:

$$
R=\frac{c_{2, f}-c_{2, i}}{c_{1, f}-c_{1, i}}
$$

where $c_{j, i}\left(c_{j, f}\right)$ indicates the initial (final) velocity of the $j$-th wave. An elastic collision corresponds to $R=1$.

\subsection{Symmetric collisions}

We first consider the collisions of two identical incoming solitary waves moving in opposite directions. Upon colliding, the waves are refracted or reflected. In some cases, the appearance of a third pulse after the collision is observed (we refer to such an event as "breather creation") as for non-exact solutions [7, 26]. Fig. 23 shows the values of the restitution coefficients together with the parameters values for which the breather creation takes place and Figs. 24 and 25, show typical examples of collisions with and without creation of additional waves. It is worthwhile to note here the high degree of elasticity of the observed dynamics, as well as the potential for inducing breather creation, especially for the higher branches and, in particular, for small values of the frequency. 

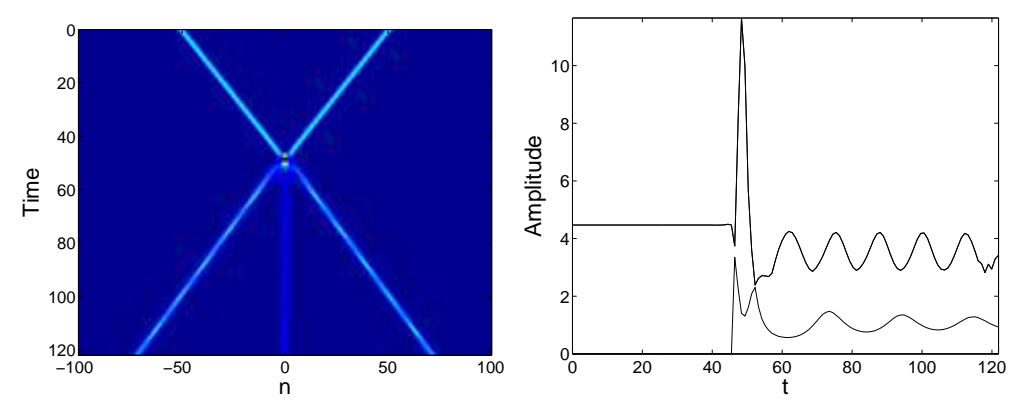

Figure 25: Collision of two waves with initial velocity $c=1.0338$ and $\Lambda=0.1129$. They are refracted / reflected after the collision with an extra breather created. The panels are similar to those of Fig. 24, but the right panel also shows the amplitude of the wave created at $n=0$.
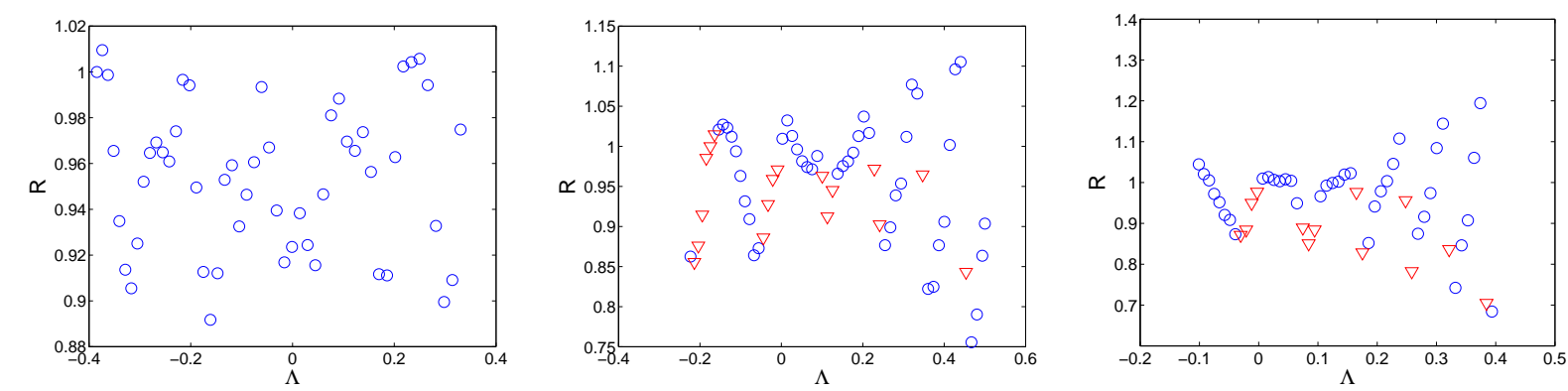

Figure 26: Dependence of the restitution coefficient for collisions of a travelling wave with $c=1.3006$ and $\Lambda=0.0143$ in branch I (left), $c=1.0338$ and $\Lambda=0.1129$ in branch II (center) and $c=0.8881$ and $\Lambda=0.0845$ in branch III (right). These collisions take place with waves of the same branch whose $\Lambda$ is indicated in the $\mathrm{X}$-axes of the graphs. Circles correspond to reflection / refraction and triangles to breather creation.

\subsection{Asymmetric collisions}

In this case, the incoming solitary waves do not have the same velocities, nor do they necessarily move in opposite directions. This allows the possibility of collisions between pulses in the same branch, as well as ones in different branches. We show in Figs. 26 the restitution coefficient for intra-band and collisions (the results are similar for inter-band collisions). Examples of collisions with and without creation are shown in Figs. 27 and 28. It is observed once again that the restitution coefficient is fairly high, while one can also note that in this case the waves are almost "transparent" (no reflection is observed); in some instances, the creation of additional waves is also observed in this setting.

The existence of solitary waves with different velocities and the same value of $\varepsilon$ allows us also to consider collisions in the same direction. The velocities of the incoming traveling waves must be very different (so that they can catch up with each other in relatively short intervals of time) and the amplitude somewhat similar (so that nontrivial collisional dynamics may ensue). These conditions are rather difficult to achieve and for this reason we restrict our considerations to a qualitative analysis of some case examples. We show in Fig. 29, two example of collisions, corresponding to reflection (there is repulsion between the waves) and refraction.

\section{Conclusions}

In this work, we have considered the issues arising when examining the existence, stability and dynamics of travelling solitary waves in a nonlinear Schrödinger lattice model with saturable nonlinearity. We have 



Figure 27: Collision of two travelling waves of different velocities. One belongs to branch I with initial velocity $c=1.3006$ and $\Lambda=0.0143$. The other one belongs to branch III, having initial velocity $c=1.0676$ and $\Lambda=-0.0751$. They are refracted / reflected after the collision. (Left) The space-time contour plot of the density is shown. (Right) The time dependence of the amplitude of the traveling waves is presented.
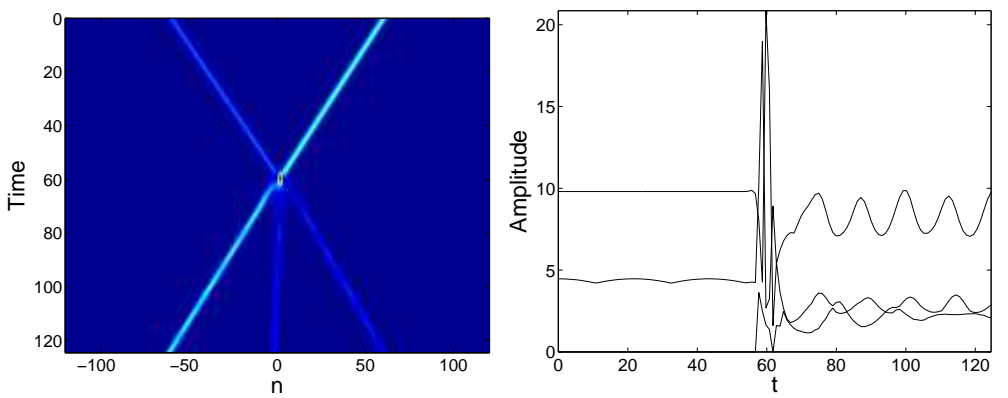

Figure 28: Collision of two waves with different velocities. One belongs to branch II with initial velocity $c=1.0338$ and $\Lambda=0.1129$. The other one belongs to branch III, having initial velocity $c=0.9876$ and $\Lambda=-0.0025$. The panels are similar to the previous figure.
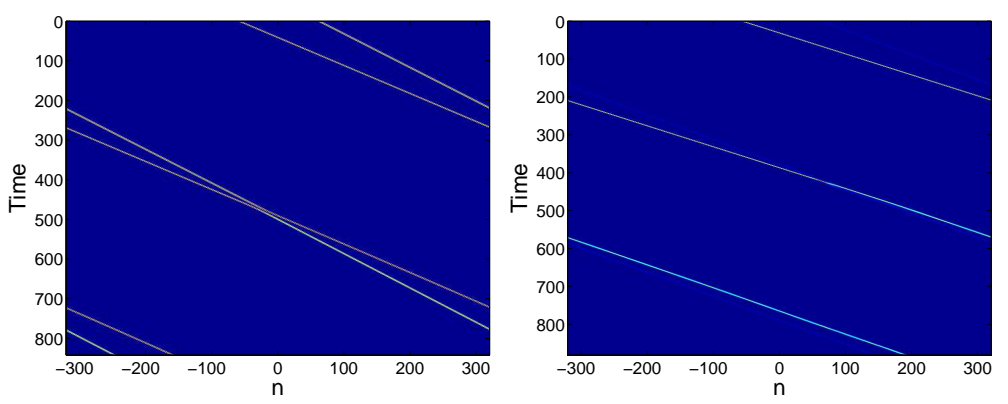

Figure 29: Collision of two traveling waves with different velocities moving in the same direction. They have (Left) $c=1.4201$ and $\Lambda=-0.3509$, belonging to branch II and $c=1.1804$ and $\Lambda=-0.1817$, belonging to branch III. The coherent structures repel each other. (Right) $c=1.6433$ and $\Lambda=-0.5597$, belonging to branch I and $c=1.1804$ and $\Lambda=-0.1817$, belonging to branch III. The waves are refracted. 
illustrated that in this context, the static problem features transparent points, that is zeros of a generalised energy barrier between site centred and intersite modes. The existence of which, loosely speaking, suggest the possibility of truly localised travelling waves with arbitrarily small wave speed. However the accumulation of linear spectrum on $c=0$ does not allow for such waves to exist without (exponentially small) oscillatory tails, that could eventually lead to radiative loss. Nevertheless, a detailed examination of the linear spectrum allows us to identify parameter regions for finite wavespeed $c$ where a single resonance of the travelling solution with plane waves occurs. Within these regions, it is possible to search for isolated, that is codimension-one parameter values where the tail of the solution vanishes. An appropriate definition of the tail based on the symmetry of the solution has been proposed and found to be particularly efficient in our numerical investigations. These isolated points lead to branches in two-parameter planes (such as speed vs. frequency or speed vs. coupling strength) which have been fully elucidated. The solutions obtained have been confirmed to travel without radiative loss in direct numerical simulations of the model. Perturbations of these embedded-soliton type solutions have been shown to lead to their "degeneration" into another element of the corresponding branch (upon suitable modification of the intrinsic frequency parameter), but clearly also illustrate the robustness of the waves. This robustness has been further confirmed by linear stability analysis and the computation of the Floquet spectra of waves. Finally, the collisions of the waves have shown a high restitution coefficient, indicating their near-elasticity. However, they also highlight the absence of integrability of the discrete model through partial amplitude decrease and phenomena such as the creation of an additional localised excitations.

A particularly interesting direction of future study would involve the examination of higher dimensional generalisations of the present work. While such generalisations are already being studied (see for instance the works of [41], [42]), demonstrating interesting possibilities about the propagation on- and off-lattice directions, there is no "numerically exact" (up to a desired accuracy) computation of travelling wave solutions nor their stability, to the best of our knowledge. Such studies are currently in progress and will be reported in future publications.

It should be stressed that while we have presented detailed numerical evidence for the existence and stability of travelling discrete solitary waves in the saturable DNLS model, we have not provided a definite mathematical proof. It would be interesting to see to what extent a rigorous analysis could be developed. Recent progress has been obtained for example by Oxtoby et al [31] by looking at the limit of small amplitude waves and computation of the so-called Stokes constant corresponding to the solutions originating at the fundamental spectral band in Figure 3. We also mention in related cubic DNLS models the work of Pelinovsky et al where a rigorous Melnikov theory is developed in a special case [32].

\section{References}

[1] M.J. Ablowitz, Z.H. Musslimani and G. Biondini, "Methods for discrete solitons in nonlinear lattices", Phys. Rev. E 65, 026602 (2002).

[2] A.A. Aigner, A.R. Champneys and V.M Rothos, "A new barrier to the existence of moving kinks in Frenkel-Kontorova lattices”, Physica D 186 148-170 (2003).

[3] S. Aubry, "Breathers in nonlinear lattices: Existence, linear stability and quantization", Physica D 103, 201, (1997);

[4] J.P. Boyd, "Weakly Nonlocal Solitary Waves and Beyond-All-Orders Asymptotics", Kluwer Academic Publishers. (1998).

[5] A.R. Champneys, J.-M Vanden-Broeck and G.J. Lord, "Do true elevation solitary waves exist? A numerical investigation”, J.Fluid Mech 454 403-417 (2002). 
[6] D. N. Christodoulides, F. Lederer and Y. Silberberg, "Discretizing light behaviour in linear and nonlinear waveguide lattices", Nature 424, 817 (2003).

[7] J. Cuevas and J.C. Eilbeck, "Discrete soliton collisions in a waveguide array with saturable nonlinearity”, Phys. Lett. A 358, 15 (2006).

[8] E.J. Doedel, A.R Champneys. T.R. Fairgrieve, Y.A. Kuznetsov, B.Sandstede \& X.J.Wang. "Auto97 continuation and bifurcation software for ordinary differential equations", ftp://ftp.es.concordia.ca/directory/doedel/auto (1997).

[9] D.B. Duncan et al., "Solitons on lattices", Physica D 68, 1 (1993);

[10] N.K. Efremidis et al., "Discrete solitons in photorefractive optically induced photonic lattices", Phys. Rev. E 66046602 (2002).

[11] S. Flach and K. Kladko, “Moving discrete breathers?”, Physica D 127 61-72 (1999).

[12] S. Flach, Y. Zolotaryuk and K. Kladko, "Moving lattice kinks and pulses: An inverse method", Phys. Rev. E 59, 6105 (1999).

[13] J.W. Fleischer, "Spatial photonics in nonlinear waveguide arrays", Opt. Express 13, 1780 (2005).

[14] J.W. Fleischer et al., "Observation of Vortex-Ring "Discrete" Solitons in 2D Photonic Lattices", Phys.Rev. Lett. 92123904 (2004).

[15] J.W. Fleischer et al., "Observation of discrete solitons in optically induced real time waveguide arrays", Phys. Rev. Lett. 90023902 (2003).

[16] J. Gómez-Gardeñes, F. Falo and L.M. Floría, "Mobile localization in nonlinear Schrödinger lattices", Phys. Lett. A 332, 213 (2004).

[17] J. Gómez-Gardeñes, L.M. Floría, M. Peyrard and A.R. Bishop, "Nonintegrable Schrödinger discrete breathers", Chaos 14, 1130 (2004).

[18] L. Hadzievski et al., "Power controlled soliton stability and steering in lattices with saturable nonlinearity", Phys. Rev. Lett. 93. 033901 (2004).

[19] M. Johansson and S. Aubry, "Growth and decay of discrete nonlinear Schrödinger breathers interacting with internal modes or standing-wave phonons", Phys. Rev. E 61, 5864, (2000)

[20] P.G. Kevrekidis, K.O. Rasmussen, and A.R. Bishop, "The Discrete Nonlinear Schrödinger Equation: A Survey of Recent Results", Int. J. Mod. Phys. B 15, 2833 (2001).

[21] P.G. Kevrekidis and D.J. Frantzeskakis, "Pattern Forming Dynamical Instabilities of Bose-Einstein Condensates", Mod. Phys. Lett. B 18, 173 (2004).

[22] Yu. S. Kivshar and G. P. Agrawal, "Optical Solitons: From Fibers to Photonic Crystals", Academic Press (San Diego, 2003).

[23] K. Kolossovski, A.R. Champneys, A.V. Buryak and R.A. Sammut, "Multi-pulse embedded solitons as bound states of quasi-solitons", Physica D 171 153-177 (2002).

[24] V.V. Konotop and V.A. Brazhnyi, "Theory of Nonlinear Matter Waves in Optical Lattices”, Mod. Phys. Lett. B 18 627, (2004). 
[25] A. Maluckov, Lj. Hadzievski and M. Stepic, "Bifurcation analysis of the localized modes dynamics in lattices with saturable nonlinearity”, Phys. D 216, 95 (2006).

[26] A. Maluckov, Lj. Hadzievski and M. Stepic, "On symmetric breather collisions in lattices with saturable nonlinearity”, Eur. Phys. J. B 53, 333 (2006).

[27] H. Martin et al., "Discrete Solitons and Soliton-Induced Dislocations in Partially Coherent Photonic Lattices”, Phys. Rev. Lett. 92123902 (2004).

[28] T.R.O. Melvin, A.R. Champneys. P.G. Kevrekidis and J. Cuevas, "Radiationless Travelling Waves in Saturable Nonlinear Schrödinger Lattices”, Phys. Rev. Lett. 97, 124101 (2006).

[29] O. Morsch and M. Oberthaler, "Dynamics of Bose-Einstein condensates in optical lattices", Rev. Mod. Phys. 78, 179 (2006).

[30] D.N. Neshev et al., "Observation of Discrete Vortex Solitons in Optically Induced Photonic Lattices", Phys. Rev. Lett. 92123903 (2004).

[31] O.F. Oxtoby and I.V. Barashenkov. "Moving solitons in the discrete nonlinear Schrödinger equation" Phys. Rev. E 76, 036603 (2007).

[32] D.E. Pelinovsky, T.R.O. Melvin and A.R. Champneys, "One-parameter localized traveling waves in nonlinear Schrödinger lattices", Preprint (2007).

[33] D.E. Pelinovsky and V.M. Rothos, "Bifurcations of travelling wave solutions in the discrete NLS equations", Physica D 202 16-36 (2005).

[34] M. Peyrard "Nonlinear dynamics and statistical physics of DNA", Nonlinearity 17, R1 (2004).

[35] M.J.D. Powell, "A hybrid method for nonlinear algebraic equations", Numerical Methods for Nonlinear Algebraic Equations. Gordon and Breach (1970).

[36] Powell Hybrid Method Code. http://www.netlib.org/minpack

[37] M. Remoissenet and M. Peyrard (eds.) "Nonlinear Coherent Structures in Physics and Biology", Springer-Verlag Berlin, (1991).

[38] M. Salerno, "Quantum deformations of the discrete nonlinear Schrödinger equation“, Phys. Rev. A 46, $6856(1992)$

[39] M. Sato, B. E. Hubbard, and A. J. Sievers "Nonlinear energy localization and its manipulation in micromechanical oscillator arrays", Rev. Mod. Phys. 78, 137 (2006).

[40] M. Stepíc et al., "One-dimensional bright discrete solitons in media with saturable nonlinearity", Phys. Rev. E 69, 066618 (2004).

[41] R.A. Vicencio and M. Johansson, "Discrete soliton mobility in two-dimensional waveguide arrays with saturable nonlinearity”, Phys. Rev. E 73, 046602 (2006).

[42] H. Susanto, P.G. Kevrekidis, R. Carretero-Gonzalez, B.A. Malomed and D.J. Frantzeskakis, "Mobility of Discrete Solitons in Quadratically Nonlinear Media”, nlin/0605048. (2006).

[43] X. Wang, Z. Chen and P.G. Kevrekidis, "Observation of Discrete Solitons and Soliton Rotation in Optically Induced Periodic Ring Lattice”, Phys. Rev. Lett. 96, 083904 (2006). 
[44] J. Yang et al., "Necklacelike Solitons in Optically Induced Photonic Lattices", Phys. Rev. Lett. 94, 113902 (2005).

[45] J. Yang, "Dynamics of Embedded Solitons in the Extended Korteweg-de Vries Equations", Stud. Appl. Maths. 106 337-365 (2001). 